\title{
Mathematical-Physical Properties of Musical Tone Systems II: Applications
}

\author{
By \\ Bruno J. Gruber \\ (Vorgelegt in der Sitzung der math.-nat. Klasse am 22. Juni 2006 \\ durch das k. M. Peter Steinhauser)
}

\begin{abstract}
In [1] mathematical-physical properties of musical tones, and musical tone systems, were discussed. In the current article the results obtained in [1] are applied to analyze tonal systems in greater detail. This is done for the case of the 3-dimensional tonal lattice system, as well as for its 2-dimensional tonal sub-lattice - the Pythagorean plane.

It will be shown that in the 3-dimensional tonal lattice space a 31-tone system can be defined which contains the familiar tone scales as substructures, namely the 12tone chromatic scales (with their subscales). Moreover, the 31-tone system contains a 3-dimensional 22/23-tone system related to a hypothetical South Indian Carnatic tone system and to the 3-tone scale of the ancient Greek Lyre.

For the case of the 2-dimensional Pythagorean sub-lattice a 29-tone system can be defined. This 29-dimensional tone lattice contains a 2-dimensional 22/23-tone system. Moreover, the 2-dimensional 29-tone system also contains the Pythagorean 12-tone systems (scales), the Pythagorean 7-tone scale and again the 3-tone scale of the ancient Greek Lyre.

Subsequently it is shown that the 2-dimensional tonal systems of the Pythagorean plane are, in fact, images of the 3-dimensional tonal systems of the 31-tonal system, if the 3 -dimensional tonal systems are projected along the Pythagorean vector $p=(-1,3,-1)$ ( $=81 / 80$, the Syntonic comma) into the Pythagorean plane. In particular, the chromatic minor scale projects upon the 12-tone Pythagorean scale, the chromatic major on another 12-tone Pythagorean scale (quite asymmetric with respect to the tone $c$ ), the 7-tone diatonic scale projects upon the 7-tone Pythagorean scale. The 22/23-tone 3-dimensional tone system consists of 11 pairs of tones, related to each other by the Pythagorean vector $p$, and the tone $c$. Each pair is mapped upon one tone in the Pythagorean plane, and these 11 images, together with the tone $c$, form the standard 12-tone Pythagorean scale. This
\end{abstract}


projection establishes not only a relationship between the tones of the 3-dimensional and 2-dimensional tonal systems, but also a functional relationship between the tone intervals of various musical systems and musical scales.

Finally, it is shown that a 3-dimensional 116-tone system exists which contains all the 3-dimensional tone systems and scales mentioned above. Moreover, it contains all the musical tones (not the overtones) of the list of tones given in [2]. As an explicit example, Table 10.1 lists all the tones, the tone sequence, and the relationship between the intervals for the various tonal systems, for the first full tone $T_{1}$ (the tone interval $c-d)$. This table also illustrates the relationship of the tones with respect to the two bases used, the (2/1), (3/2) and (5/3) basis used in this article (and in [1]), and the basis $(2 / 1),(5 / 4)$ and $(3 / 2)$ basis used in [2].

The reversal of certain tone sequences of the images, due to the map along the Pythagorean vector $p$, is also discussed, as well as a special form for the formula for the intervals between the tones derived which expresses the intervals as a linear equation in terms of three discrete parameters.

\section{Introduction}

Tonal scales and tonal systems will be discussed in this article. Tonal systems, as distinguished from tonal scales, are defined as ordered inventories (Tonmaterial) of musical tones from which tones for actual musical scales can be selected, as for example the tonal system obtained by RIEMANN [2]. The ordering of the tonal inventory is however not merely an ordering according to frequency (a one-parameter ordering) but an ordering according to a lattice structure (a three-parameter ordering), of the kind used by MAZzOLA [3]. While the analysis given in this article is strictly restricted to "lattice properties" of tonal lattice systems there are obvious implications for actual musical scales [3], [4].

The musical tones are defined by ratios of frequencies $\nu / \nu_{0}$, whereby $\nu_{0}$ is an arbitrary, but fixed reference tone. The frequencies $\nu$ within the $n$-th octave can be expressed in the form

$$
\nu=\nu_{0} 2^{n}(1+\delta / 2 \pi), \quad 0 \leq \delta / 2 \pi \leq 1, \quad n=0, \pm 1, \pm 2, \pm 3, \ldots
$$

or in the form

$$
\nu=\nu_{0} 2^{n+\xi / 2 \pi,} \quad 0 \leq \xi / 2 \pi \leq 1, \quad n=0, \pm 1, \pm 2, \pm 3, \ldots
$$

with

$$
\xi / 2 \pi=\log _{2}(1+\delta / 2 \pi) .
$$

The octave tones, given by the parameter value $\delta / 2 \pi=0$, are

$$
\nu=\nu_{0}^{n}=\nu_{0} 2^{n}, \quad \delta / 2 \pi=0, \quad n=0, \pm 1, \pm 2, \pm 3, \ldots
$$


The upper index $n$ in the expression $\nu^{n}$ for the frequency $\nu$ denotes the $n$-th octave. (This notation for the $n$-th octave will also hold for any alphabetical musical tone like the tone $a^{n}$, in distinction to $(a)^{n}$ which denotes the $n$-th power of the frequency ratio $\nu=5 / 3$ associated with the tone $a$ ). The $n=0$ octave is called the basic octave. In this article the value chosen for $\nu_{0}$ is $\nu_{0}=c$, where $c$ denotes the first tone of the diatonic scale of $C$-major. Thus for the case $n=0$ holds

$$
\nu_{0}=\nu^{0}=c .
$$

That is, the reference frequency $\nu_{0}$ is chosen to be the first tone of the $n=0$ octave which has the alphabetical name $c$.

Note that the expression given by Eq. (1.1) is the inverse function to the logarithmic function

$$
\log _{2}\left(\nu / \nu_{0}\right)=n+\log _{2}(1+\delta / 2 \pi)=n+\xi / 2 \pi .
$$

Thus both expressions, Eqs. (1.1) and (1.3), carry the same information. Eq. (1.3) is essentially an expression for the distance, in cent, of the tone $\nu$ from the reference tone $\nu_{0}$ (apart from a factor 1,200).

The relationship Eq. (1.1c) between frequency and perception (Hörempfinden) has also been discussed in ref. [5].

The musical tones considered in this article (and in refs. [1] and [2]) are of the form

$$
\begin{gathered}
\nu / \nu_{0}=(n, m, r)=(2 / 1)^{n}(3 / 2)^{m}(5 / 3)^{r}, \\
n, m, r=0, \pm 1, \pm 2, \pm 3 \ldots
\end{gathered}
$$

It then holds for the musical tones $\nu / \nu_{0}$ which are contained within the basic (2/1)-octave, that

$$
\begin{gathered}
1 \leq(2 / 1)^{n}(3 / 2)^{m}(5 / 3)^{r}=(n, m, r) \leq 2, \\
n, m, r=0, \pm 1, \pm 2, \pm 3 \ldots
\end{gathered}
$$

It was shown in ref. [1] that the musical tones $(n, m, r)$, Eq. (1.4), can be mapped onto lattice points of a 3-dimensional scaled lattice, with the distances between the lattice points scaled by the factor $(2 / 1)^{n}$ along the $n$-axis, by the factor $(3 / 2)^{m}$ along the $m$-axis, and by the factor $(5 / 3)^{r}$ along the $r$-axis. That is, each lattice point $(n, m, r)$ represents a musical tone corresponding to the frequency $\nu / \nu_{0}$, as defined by Eq. (1.4).

Thus, instead of a single number $\nu / \nu_{0}$ representing a musical tone, the musical tones are now expressed in terms of three discrete parameters. The properties of the 3-dimensional lattice associated with the musical tones provide a mathematical structure which is not available if the musical tones are merely given in terms of pure numbers $\nu / \nu_{0}$ 
(which now represents only one property of a musical tone $(n, m, r)$, namely "its distance" from the lattice origin). This additional mathematical structure of the musical tones $(n, m, r)$ permits new insights into the structure of musical tones, and moreover supplies the mathematical tools needed to systematically study musical tone systems.

As it was noted above, the lattice point (vector) $(n, m, r)$ represents a musical tone in a 3-dimensional lattice space, and with this lattice point $(n, m, r)$ is associated a numerical value, namely the number $\nu / \nu_{0}$ (the frequency ratio). In this article a musical tone $(n, m, r)$ will be understood to represent simultaneously a 3-dimensional vector, and its associated numerical value $\nu / \nu_{0}$. Thus, symbols like $s_{1}, s_{2}, s_{3}$, representing musical intervals, denote simultaneously vectors and their associated numerical value,

$$
\begin{aligned}
& s_{1}=\nu_{1} / \nu_{0}=\left(n_{1}, m_{1}, r_{1}\right), \\
& s_{2}=\nu_{2} / \nu_{0}=\left(n_{2}, m_{2}, r_{2}\right), \\
& s_{3}=\nu_{3} / \nu_{0}=\left(n_{3}, m_{3}, r_{3}\right) .
\end{aligned}
$$

It is then the mathematical operation used which will distinguish between the two meanings, namely

$$
s_{1}+s_{2}=s_{3}
$$

is understood to be equal to the vector sum of $s_{1}$ and $s_{2}$,

$\left(n_{1}, m_{1}, r_{1}\right)+\left(n_{2}, m_{2}, r_{2}\right)=\left(n_{1}+n_{2}, m_{1}+m_{2}, r_{1}+r_{2}\right)=\left(n_{3}, m_{3}, r_{3}\right)$,

while the equation

$$
s_{1} s_{2}=s_{3}
$$

is understood to be equal to the ordinary product of the frequencies associated with $s_{1}$ and $s_{2}$,

$$
\left(\nu_{1} / \nu_{0}\right)\left(\nu_{2} / \nu_{0}\right)=\left(\nu_{3} / \nu_{0}\right), \quad \nu_{0}=1 \text {. }
$$

The musical tone systems, i.e. the various musical scales, form subsets of lattice points within the 3-dimensional lattice space [1]. For a set of lattice points to form a musical scale certain conditions apply. In particular the "closure condition" applies. That is, starting out with a tone $\nu_{0} / \nu_{0}=1=(0,0,0)$ the octave tone $2 \nu_{0} / \nu_{0}=2=(1,0,0)$ must be reached in an integer number of (discrete) steps - the intervals between the tones. Thus, once the various intervals - interval vectors - have been chosen, the lattice properties will determine the possible musical scales which can be based upon the chosen interval vectors. The vector sum of vector-intervals, however, must not only reach the octave lattice point but must do this in such a manner that in 
each step only musical tones of the basic $n=0$ octave are reached, Eqs. (1.5) and (1.6a).

Using Eq. (1.5) it is possible to determine all musical tones $\nu / \nu_{0}=$ $(n, m, r)$ which belong to the basic $n=0$ octave $[0,1]$. Choosing from among these musical tones certain tones as interval vectors,

$$
\left(n_{1}, m_{1}, r_{1}\right),\left(n_{2}, m_{2}, r_{2}\right),\left(n_{3}, m_{3}, r_{3}\right), \ldots
$$

it must hold

$$
\begin{gathered}
(0,0,0)=1 \leq(0,0,0)+k_{1}\left(n_{1}, m_{1}, r_{1}\right)+k_{2}\left(n_{2}, m_{2}, r_{2}\right) \\
+k_{3}\left(n_{3}, m_{3}, r_{3}\right)+\cdots \leq 2=(1,0,0) \\
k_{i}=0,1,2, \ldots, k_{i}^{\max }, \quad i=1,2,3, \ldots
\end{gathered}
$$

and

$$
\begin{gathered}
(0,0,0)+k_{1}^{\max }\left(n_{1}, m_{1}, r_{1}\right)+k_{2}^{\max }\left(n_{2}, m_{2}, r_{2}\right) \\
+k_{3}^{\max }\left(n_{3}, m_{3}, r_{3}\right)+\cdots=(1,0,0) .
\end{gathered}
$$

The number of tones $N$ of the musical system/scale is then given by

$$
N=k_{1}^{\max }+k_{2}^{\max }+k_{3}^{\max }+\cdots .
$$

The formulas given by Eq. (1.6) do not determine the order of the intervals, i.e. the sequence of the tones, nor the tones themselves. The tone sequence is only partially obtained by the requirement of a monotonic increase of the interval sequence. However, since there are only two fundamental intervals $s_{1}$ and $s_{2}$ for the 2-dimensional musical scales, and since moreover the Pythagorean musical scale is assumed to form a subscale of any larger 2-dimensional musical system, the sequence for the interval-vectors $s_{1}$ and $s_{2}$ is to some extent determined. The 31-tone, 3-dimensional musical scale, is based upon three fundamental constants, namely $S_{1}$ and $S_{2}$ and $p=(-1,3,-1)=81 / 80$. Relationships obtained through the lattice properties then show that the constants $S_{1}$ and $S_{2}$ are expressible in terms of $s_{1}, s_{2}$ and $p$. This limits the possible choice of tones for 3 -dimensional musical systems if the images of the 3-dimensional tones, projected along the constant vector $p$, are to be tones of musical systems of the 2-dimensional Pythagorean plane. The unique functional relationship between the intervals $\left\{s_{1}, s_{2}\right\}$ and $\left\{S_{1}, S_{2}, p\right\}$, given by Eqs. (6.3) and (6.4), together with the required simultaneous consistency of both musical systems within the constraints of the musical tone lattice, permits the selection of a tone system for a 31-tone 3-dimensional musical system from among the 3-dimensional tones projected along the vector $p$ onto a 29-tone 2-dimensional tone system. 
In [1] it was found that the Pythagorean musical scale is contained in a 2-dimensional sub-lattice

$$
(n, m, 0)=(n, m), \quad n, m=0, \pm 1, \pm 2, \pm 3, \ldots
$$

of the 3-dimensional musical lattice

$$
(n, m, r), \quad n, m, r=0, \pm 1, \pm 2, \pm 3, \ldots
$$

The Greek Pythagorean musical scale, dating back to around 500 BCA appears to have been predated, by about 100 years, by similar musical relationships in Chinese musical theory, dating back to 600 BCA [6]. This may indicate some kind of intercultural dissemination of musical knowledge between ancient cultures, or may have been simply the result of rational reasoning inherent in human nature. This reasoning may not be limited to these two cultures but may include the South Indian Dravidian culture, whose music tradition, Carnatic music, dates back to before 1,000 BCA [7]. Using this reasoning as a work hypothesis, it may be possible that the musical traditions of these cultures may have been related, or been mutually influenced, and were possibly at some time all based on the 2-dimensional Pythagorean musical lattice system, while the 3-dimensional musical lattice system may have been a later western development. This reasoning underlies the investigation of the tonal systems of the Pythagorean(-Carnatic) plane.

Applying Eq. (1.5) to the Pythagorean(-Carnatic) plane it is found that there exist two tone intervals (vectors) $\left\{s_{1}, s_{2}\right\}$ such that a tonal system of 29 tones can be constructed. These two fundamental tones $\left\{s_{1}, s_{2}\right\}$ will be called srutis since they compare closely to the intervals assumed for the srutis of the Carnatic music [7]. It will then be shown that the 29-tone system contains as subsystems a 2-dimensional 23-tone and 22-tone sub-system. These two sub-systems are based upon three intervals $\left\{s_{1}, s_{2}, s_{3}=\left(s_{1} s_{2}\right)\right\}$, with $s_{3}$ not independent, but given in terms of $s_{1}$ and $s_{2}$. Another tonal subsystem is given by a set of 17 tones of the 29-tone tone system. These 17 tones of the 2-dimensional Pythagorean lattice are the images of 17 tones of the 3-dimensional tone lattice system, consisting of the tones corresponding to the 7 natural diatonic tones and the 10 tones of the sharps and flats of the chromatic scales. This subsystem is based upon the two intervals $\left\{\left(s_{1} s_{3}\right), s_{3}\right\}$. The Pythagorean 7-tone scale is also based upon two intervals, namely $\left\{\left(s_{1}^{3} s_{2}^{2}\right), s_{3}\right\}$, while the 3-tone scale of the ancient Greek Lyre is based upon the two intervals $\left\{\left(s_{1}^{7} s_{2}^{5}\right),\left(s_{1}^{3} s_{2}^{2}\right)\right\}$. (A parenthesis around an expression indicates that the expression acts as a unit.)

The question then arises in which manner the 2-dimensional tone systems are related to the western culture 3-dimensional tone 
systems. As it was mentioned above, while only two fundamental constants $\left\{s_{1}, s_{2}\right\}$ are needed for the musical scales in the Pythagorean plane, three fundamental constants $\left\{S_{1}, S_{2}, p\right\}$ are needed for the 3-dimensional musical tone systems. Moreover, it was also mentioned before, that the third constant, the vector $p=(-1,3,-1)=81 / 80$, known as the Syntonic comma, defines a map (projection) from the 3-dimensional 31-tone lattice into the 2-dimensional Pythagorean tonal plane. All tones of the 3-dimensional lattice lying on a line, defined by the vector $p$, are projected upon the same 2-dimensional tone. This includes, in particular also the tones and the interval factors of the standard 3-dimensional chromatic musical scales. This results in a unique functional relationship between the fundamental intervals of both systems, the properties of the 3-dimensional musical scales being reflected in the 2-dimensional musical scales, and vice versa.

The mapping from the 3-dimensional musical lattice space into the 2-dimensional Pythagorean lattice subspace is given by the following equation: All 3-dimensional musical lattice points

$$
(n+r, m-3 r, r), \quad r=0, \pm 1, \pm 2, \pm 3, \ldots,
$$

are mapped, along the vector $p$, onto the single 2-dimensional tone

$$
\begin{gathered}
(n, m, 0)=(n+r, m-3 r, r)+r(-1,3,-1), \\
r=0, \pm 1, \pm 2, \pm 3, \ldots .
\end{gathered}
$$

The inverse process represents an embedding of a 2-dimensional lattice tone system into the 3-dimensional lattice tone system and is obviously not unique. The ambiguity for the embedding can be resolved by the requirement of simultaneous consistency of the 3-dimensional tone system and its mapped image in the Pythagorean plane. The 3-dimensional tone system can then be considered to represent a consistent embedding of the 2-dimensional tone systems into the 3-dimensional lattice space.

The 3-dimensional tonal lattice system with lattice points

$$
(n, m, r), \ldots \quad n, m, r=0, \pm 1, \pm 2, \pm 3, \ldots,
$$

can be considered to be made up of a set of Pythagorean planes, each plane labeled by $r$,

$$
r=0, \pm 1, \pm 2, \pm 3, \ldots
$$

such that for each fixed value $r$

$$
(n, m, r) \quad n, m=0, \pm 1, \pm 2, \pm 3, \ldots
$$

represents a separate Pythagorean plane.

These Pythagorean planes can be considered to be related to each other by translations $p=(-1,3,-1)$ such that the origin of the $r=0$ 
Pythagorean plane - the tone $c=(0,0,0)$ - is translated into the tone corresponding to the (new) origin of the $r$-th Pythagorean plane - the tone $r(-1,3,-1), \quad r=0, \pm 1, \pm 2, \pm 3, \ldots$ These Pythagorean planes will be called equivalent with respect to the Pythagorean vector $p$. Similarly, musical tones related by multiples of the Pythagorean vector $p$ will be called equivalent with respect to translations by the Pythagorean vector $p=(-1,3,-1)$.

In what follows the names for the musical intervals/tones are taken from the "List of Intervals" given in ref. [8].

\section{The Two-Dimensional Lattice Tone System}

In this section the 29-tone 2-dimensional system will be derived. The tones of this system lie all in the Pythagorean plane,

$$
\begin{gathered}
\nu / \nu_{0}=(n, m, r=0)=(2 / 1)^{n}(3 / 2)^{m}, \\
n, m=0, \pm 1, \pm 2, \pm 3, \ldots
\end{gathered}
$$

The lattice points given by Eq. (2.1) correspond to the musical lattice tones as defined in [1]. Thus the musical lattice tones form a 2-dimensional scaled sub-lattice given by

$$
\begin{gathered}
\nu / \nu_{0}=(n, m, 0)=n(1,0,0)+m(0,1,0), \\
m, r=0, \pm 1, \pm 2, \pm 3, \ldots
\end{gathered}
$$

or for short,

$$
\nu / \nu_{0}=(n, m)=n(1,0)+m(0,1), \quad m, r=0, \pm 1, \pm 2, \pm 3, \ldots
$$

Therefore, these musical tones are uniquely characterized as lattice points (a two-parameter object)

$$
(n, m),
$$

having associated with them the numerical value (a one-parameter object),

$$
\nu / \nu_{0}=(2 / 1)^{n}(3 / 2)^{m} .
$$

Eq. (2.5) represents the frequency ratio of the musical tone $(n, m)$.

By standard convention, the numerical values $\nu / \nu_{0}$ for the musical tones of an octave are given alphabetic letter names like, for example, the letters $c, d, e, f, g, a, h(b),\left(c^{1}\right)$, for the 7 tones of the natural diatonic musical scale. In order to distinguish between the musical tones of the 3-dimensional lattice space and the subsystem of tones of 
the 2-dimensional Pythagorean lattice space, the tones of the latter will be characterized by a bar over the alphabetic letter,

$$
\bar{c}(=c), \quad \bar{d}(=d), \quad \bar{e}, \quad \bar{f}(=f), \quad \bar{g}(=g), \quad \bar{a}, \quad \bar{h}, \quad \bar{c}^{1}\left(=c^{1}\right) .
$$

This convention is made in order that the alphabetical symbols, representing tones of the two systems which are related by the Pythagorean vector, correspond to each other, i.e. a tone with a bar, like the tone $\bar{a}$ of the Pythagorean musical scale, is the image of the 3-dimensional lattice tone without a bar, namely the image of the tone $a$ of the natural diatonic musical scale.

The ratio of two frequency ratios corresponding to two musical tones $\nu_{1} / \nu_{0}$ and $\nu_{2} / \nu_{0}$ (the difference, if considered as vectors),

$$
I_{12}=\nu_{2} / \nu_{1},
$$

is called an interval factor (interval vector) $I_{12}$. In order to construct a musical system it is necessary, as a first step, to find interval vectors which satisfy Eq. (1.6).

This is done by inserting, consecutively, values $n, m$ into the inequality, Eq. (1.5),

$$
1 \leq(n, m, 0)=(2 / 1)^{n}(3 / 2)^{m} \leq 2, \quad n, m=0, \pm 1, \pm 2, \pm 3, \ldots,
$$

and then rejecting those tonal intervals $(n, m, 0)$ which do not satisfy the inequality. In this manner intervals are obtained which may be suitable for a tonal system. It is found that the two intervals

$$
\begin{aligned}
s_{1}= & (-7,12,0)=3^{12} / 2^{19}=531,441 / 524,288=1.01364326, \\
& (\text { Pythagorean comma) } \\
s_{2}= & (10,-17,0)=2^{27} / 3^{17}=134,217,728 / 129,140,163 \\
= & 1.03931825, \quad \text { (Pyth. double diminished 3rd })
\end{aligned}
$$

satisfy the closure condition

$$
s_{1}^{17} s_{2}^{12}=2, \quad N=29,
$$

for a 29-tone musical tone system. The cyclic ordering of these intervals, given by

$$
\begin{aligned}
& \left|s_{1}, s_{2}, s_{1}, s_{2}, s_{1}\right| s_{1}, s_{2}, s_{1}, s_{2}, s_{1}\left|s_{1}, s_{2}, s_{1}, s_{2}, s_{1}\right| \\
& \left|s_{1}, s_{2}, s_{1}, s_{2}, s_{1}\right| s_{1}, s_{2}, s_{1}, s_{2}, s_{1} \mid s_{1}, s_{2}, s_{1}, s_{2},
\end{aligned}
$$




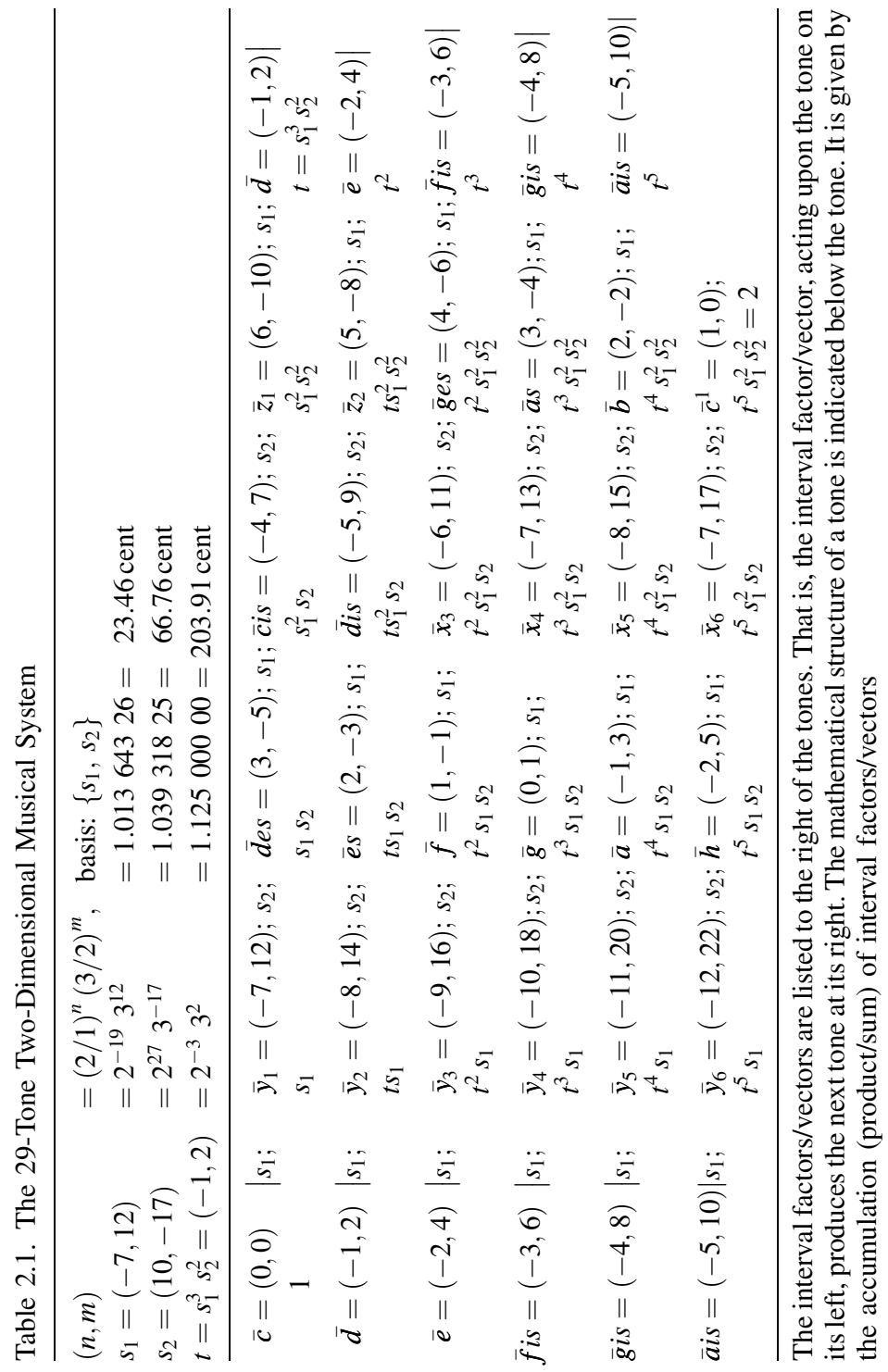


Table 2.2. The Interval Vectors and the Interval Factors for the Two-Dimensional 29-Tone Musical System

$s_{1}=(-7,12)=1.013643=23.46$ cent
$s_{2}=(10,-17)=1.039318=66.76$ cent

The tone interval $\boldsymbol{l}\left(\left(n_{1}, m_{1}\right),\left(n_{2}, m_{2}\right)\right)$ between two tones $\left(n_{1}, m_{1}\right)$ and $\left(n_{2}, m_{2}\right)$ is given, in terms of cents, by the formula, Eq. (9.9):

\begin{tabular}{|c|c|c|c|c|}
\hline $\bar{c}=$ & & $(0,0)=$ & $2^{0} 3^{0}=$ & 1.000000 \\
\hline $\bar{y}_{1}=$ & $s_{1}=$ & $(-7,12)=$ & $2^{-19} 3^{12}=$ & $1.013643\left(=s_{1}\right)$ \\
\hline $\bar{d} e s=$ & $s_{1} s_{2}=$ & $(3,-5)=$ & $2^{8} 3^{-5}=$ & $1.053498\left(=s_{3}=s_{1} s_{2}\right)$ \\
\hline $\bar{c} i s=$ & $s_{1}^{2} s_{2}=$ & $(-4,7)=$ & $2^{-11} 3^{7}=$ & 1.067871 \\
\hline $\bar{z}_{1}=$ & $s_{1}^{2} s_{2}^{2}=$ & $(6,-10)=$ & $2^{16} 3^{-10}=$ & 1.109858 \\
\hline $\bar{d}=$ & $s_{1}^{3} s_{2}^{2}=$ & $(-1,2)=$ & $2^{-3} 3^{2}=$ & 1.125000 \\
\hline $\bar{y}_{2}=$ & $s_{1}^{4} s_{2}^{2}=$ & $(-8,14)=$ & $2^{-22} 3^{14}=$ & 1.140348 \\
\hline $\bar{e} s=$ & $s_{4}^{4} s_{2}^{3}=$ & $(2,-3)=$ & $2^{5} 3^{-3}=$ & 1.185185 \\
\hline $\bar{d} i s=$ & $s_{1}^{5} s_{2}^{3}=$ & $(-5,9)=$ & $2^{-14} 3^{9}=$ & 1.201355 \\
\hline $\bar{z}_{2}=$ & $s_{1}^{5} s_{2}^{4}=$ & $(5,-8)=$ & $2^{13} 3^{-8}=$ & 1.248590 \\
\hline $\bar{e}=$ & $s_{1}^{6} s_{2}^{4}=$ & $(-2,4)=$ & $2^{-6} 3^{4}=$ & 1.265625 \\
\hline $\bar{y}_{3}=$ & $s_{1}^{7} s_{2}^{4}=$ & $(-9,16)=$ & $2^{-25} 3^{16}=$ & 1.282892 \\
\hline $\bar{f}=$ & $s_{1}^{7} s_{2}^{5}=$ & $(1,-1)=$ & $2^{2} 3^{-1}=$ & 1.333333 \\
\hline $\bar{x}_{3}=$ & $s_{1}^{8} s_{2}^{5}=$ & $(-6,11)=$ & $2^{-17} 3^{11}=$ & 1.351524 \\
\hline $\bar{g} e s=$ & $s_{1}^{8} s_{2}^{6}=$ & $(4,-6)=$ & $2^{10} 3^{-6}=$ & 1.404664 \\
\hline $\bar{f} i s=$ & $s_{1}^{9} s_{2}^{6}=$ & $(-3,6)=$ & $2^{-9} 3^{6}=$ & 1.423828 \\
\hline $\bar{y}_{4}=$ & $s_{1}^{10} s_{2}^{6}=$ & $(-10,18)=$ & $2^{-28} 3^{18}=$ & 1.443157 \\
\hline $\bar{g}=$ & $s_{1}^{10} s_{2}^{7}=$ & $(0,1)=$ & $2^{-1} 3^{1}=$ & 1.500000 \\
\hline $\bar{x}_{4}=$ & $s_{1}^{11} s_{2}^{7}=$ & $(-7,13)=$ & $2^{-20} 3^{13}=$ & 1.520465 \\
\hline $\bar{a} s=$ & $s_{1}^{11} s_{2}^{8}=$ & $(3,-4)=$ & $2^{7} 3^{-4}=$ & 1.580025 \\
\hline $\bar{g} i s=$ & $s_{1}^{12} s_{2}^{8}=$ & $(-4,8)=$ & $2^{-12} 3^{8}=$ & 1.601807 \\
\hline $\bar{y}_{5}=$ & $s_{1}^{13} s_{2}^{8}=$ & $(-11,20)=$ & $2^{-31} 3^{20}=$ & 1.623660 \\
\hline $\bar{a}=$ & $s_{1}^{13} s_{2}^{9}=$ & $(-1,3)=$ & $2^{-4} 3^{3}=$ & 1.687500 \\
\hline $\bar{x}_{5}=$ & $s_{1}^{14} s_{2}^{9}=$ & $(-8,15)=$ & $2^{-23} 3^{15}=$ & 1.710523 \\
\hline $\bar{b}=$ & $s_{1}^{14} s_{2}^{10}=$ & $(2,-2)=$ & $2^{4} 3^{-2}=$ & 1.777777 \\
\hline $\bar{a} i s=$ & $s_{1}^{15} s_{2}^{10}=$ & $(-5,10)=$ & $2^{-15} 3^{10}=$ & 1.802032 \\
\hline $\bar{y}_{6}=$ & $s_{1}^{16} s_{2}^{10}=$ & $(-12,22)=$ & $2^{-34} 3^{22}=$ & 1.862618 \\
\hline $\bar{h}=$ & $s_{1}^{16} s_{2}^{11}=$ & $(-2,5)=$ & $2^{-7} 3^{5}=$ & 1.898437 \\
\hline $\bar{x}_{6}=$ & $s_{1}^{17} s_{2}^{11}=$ & $(-9,17)=$ & $2^{-26} 3^{17}=$ & 1.924338 \\
\hline $\bar{c}^{1}=$ & $s_{1}^{17} s_{2}^{12}=$ & $(1,0)=$ & $2^{1} 3^{0}=$ & 2.000000 \\
\hline
\end{tabular}


yields a musical system. Calculation of the cent (out of 1,200 cent) yields the values

$$
\begin{aligned}
& s_{1}=23.46 \text { cent }, \\
& s_{2}=66.76 \text { cent } .
\end{aligned}
$$

A third interval factor, to be of importance later on, is given by

$$
s_{3}=s_{1} s_{2}\left(=s_{1}+s_{2} \text {, in vector form }\right)=90.22 \text { cent }(\text { Limma }) \text {. }
$$

These values correspond closely to the approximate values of the sruti (interval factors/vectors) for a hypothetical 22-tone (or 23-tone) Carnatic musical system, given in [7], namely

$$
s_{1}=22 \text { cent }, \quad s_{2}=66 \text { cent }, \quad \text { and } \quad s_{3}=90 \text { cent. }
$$

Thus the three interval factors $s_{1}, s_{2}, s_{3}$ will be referred to as sruti.

It is then seen that a 29-tone musical system is obtained, with the closure condition (2.9), and is given in vector form, by

$$
17(-7,12)+12(10,-17)=(1,0)=\bar{c}^{1}=c^{1}
$$

or equivalently by

$$
s_{1}^{17} s_{2}^{12}=2 .
$$

This implies that the tone $c_{0}\left(=c^{0}\right.$, with upper index $\left.n=0\right)$ is "musically equivalent" to the tone $c^{1}$ (though not identical to $c^{1}$ ), but differing from $c_{0}$ by the scaling factor 2 , with the next octave "cycle" starting with the tone $c^{1}$.

Thus there exists a cycle of 29 tonal steps (a sequence of sruti $s_{1}$ and $s_{2}$ ) such that the sequence "closes" (i.e. the tone $c^{1}$ is reached, the "octet condition"). Moreover it holds that

$$
\begin{aligned}
& 3(-7,12)+2(10,-17)=(-1,2)=\bar{d}=d, \\
& s_{1}^{3} s_{2}^{2}=9 / 8 \\
& 2(3(-7,12)+2(10,-17))=(-2,4)=\bar{e}=e+p, \\
& \left(s_{1}^{3} s_{2}^{2}\right)^{2}=81 / 64=(5 / 4)(81 / 80)=e \cdot p, \\
& 3(3(-7,12)+2(10,-17))=(-3,6)=\bar{f} i s=f i s+2 p, \\
& \left(s_{1}^{3} s_{2}^{2}\right)^{3}=729 / 512=(25 / 18)(81 / 80)^{2}=(f i s) \cdot p^{2}, \\
& 4(3(-7,12)+2(10,-17))=(-4,8)=\bar{g} i s=g i s+2 p, \\
& \left(s_{1}^{3} s_{2}^{2}\right)^{4}=6,561 / 4,096=(25 / 16)(81 / 80)^{2}=(\text { gis }) \cdot p^{2}
\end{aligned}
$$




$$
\begin{aligned}
& 5(3(-7,12)+2(10,-17))=(-5,10)=\bar{a} i s=a i s+3 p \\
& \left(s_{1}^{3} s_{2}^{2}\right)^{5}=59,049 / 32,768=(125 / 72)(81 / 80)^{3}=(\text { ais }) \cdot p^{3} \\
& 5(3(-7,12)+2(10,-17))+2(-7,12)+2(10,-17) \\
& \quad=(1,0,0)=\bar{c}^{1}=c^{1} \\
& \left(s_{1}^{3} s_{2}^{2}\right)^{5}\left(s_{1}^{2} s_{2}^{2}\right)=2=\bar{c}^{1}=c^{1} .
\end{aligned}
$$

The 29-tone 2-dimensional musical system is given in Table 2.1. The tones $\bar{x}, \bar{y}, \bar{z}, \bar{w}$ listed in this table appear not to have standardized names. It will be noted that this table exhibits a great amount of symmetry. Table 2.2 lists the numerical values for the tones of the 29-tone musical system.

\section{The Two-Dimensional 23/22 Musical Tone Systems}

A 22/23-tone system can be obtained from the 29-tone system, discussed above, by choosing three distinct intervals, namely three sruti. Choosing the two independent sruti, $s_{1}$ and $s_{2}$, and forming a third sruti, $s_{3}=\left(s_{1} s_{2}\right)$, the set of three sruti

$$
\left\{s_{1}, s_{2}, s_{3}=\left(s_{1} s_{2}\right)\right\}
$$

forms the intervals for the 22/23-tone musical system.

It might be remarked that, if the Carnatic musical scale should have been a Pythagorean type scale, the uncertainty concerning the actual number of tones of the Carnatic musical scale, 22 tones or more [7], or denying the existence of a Carnatic musical scale [9], may have to do with the fact that out of the 29-tone 2-dimensional musical system at different times different numbers of tones were selected to form different Carnatic tone systems.

The sequence of intervals for the Carnatic scales/systems is given by

$$
\begin{aligned}
& \left|s_{3}, s_{1}, s_{2}, s_{1}\right| s_{3}, s_{1}, s_{2}, s_{1}\left|s_{3}, s_{1}, s_{2}, s_{1}\right| \\
& \left|s_{3}, s_{1}, s_{2}, s_{1}\right| s_{3}, s_{1}, s_{2}, s_{1} \mid s_{3}, s_{1}, s_{2}, \quad N=23 \text { tones, }
\end{aligned}
$$

or

$$
\begin{aligned}
& \left|s_{3}, s_{1}, s_{2}, s_{1}\right| s_{3}, s_{1}, s_{2}, s_{1}\left|s_{3}, s_{1}, s_{2}, s_{1}\right| \\
& \left|s_{3}, s_{1}, s_{2}, s_{1}\right| s_{3}, s_{1}, s_{2}, s_{1} \mid s_{3}, s_{3}, \quad N=22 \text { tones } \\
& t=s_{3} s_{1}^{2} s_{2}=(-1,2)=9 / 8=\bar{d}=d .
\end{aligned}
$$

It will be noted that the sequence of sharps and flats in the Carnatic scale, like $\bar{d} e s$ and $\bar{c} i s$, is reversed from the sequence of sharp and flats des and cis, in the chromatic scales. This is caused by the prop- 
B. J. Gruber

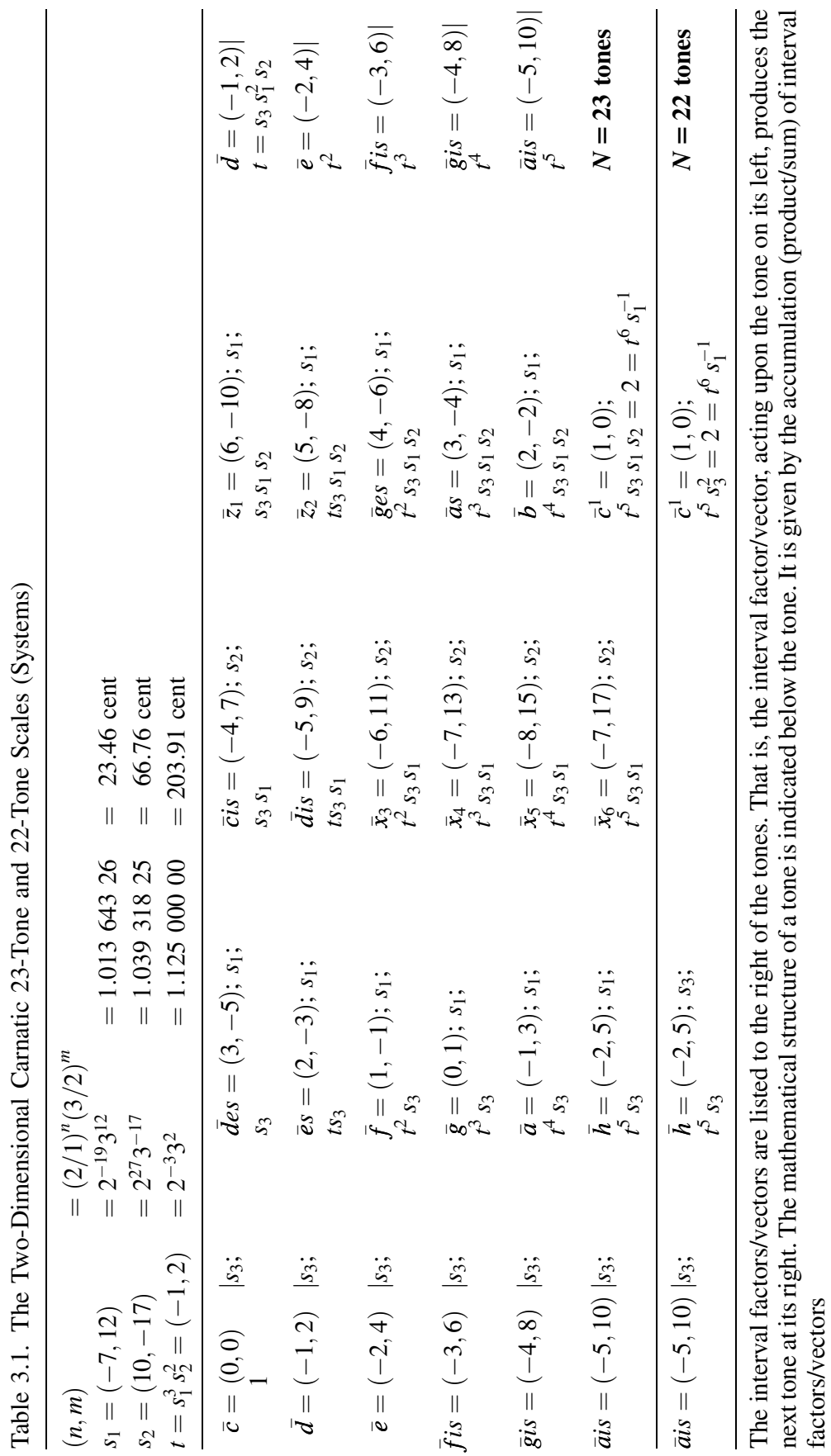


erty of the map, along the Pythagorean vector $p=(-1,3,-1)=81 / 80$, from the (scaled) chromatic lattice into the (scaled) Pythagorean plane (underlying nonlinear properties of the tonal lattice). For details on the structure of tones and the intervals see Table 3.1.

\section{Scales Contained in the Two-Dimensional 23/22 Musical Tone System}

In this section some of the musical systems/scales will be discussed which can be derived from the Carnatic musical system. The scales/ systems discussed are

(1) A 17-tone system consisting of the images in the Pythagorean plane of the set of the 7 natural diatonic tones, the 5 sharps and the 5 flats, see Sect. 7;

(2) two 12-tone Pythagorean scales;

(3) the Pythagorean musical 7-tone scale, and

(4) the 3-tone scale of the ancient Greek Lyre.

A summary for these musical scales can be found in Table 4.1.

(1) The 17-tone system is obtained from the 22-tone Carnatic system/scale by choosing the two interval factors as

$$
\left\{s_{1}, s_{3}=\left(s_{1} s_{2}\right)\right\}
$$

and choosing the interval sequence

$$
\begin{aligned}
& \left|s_{3}, s_{1}, s_{3}\right| s_{3}, s_{1}, s_{3}\left|s_{3}, s_{3}, s_{1}\right| \\
& \left|s_{3}, s_{3}, s_{1}\right| s_{3}, s_{3}, s_{1} \mid s_{3}, s_{3}, \quad N=17 \text { tones. }
\end{aligned}
$$

Thus this system depends on the two sruti $s_{1}$ and $s_{3}$ only.

(2) The Pythagorean 12-tone scales are based upon the interval factors

$$
\left\{\left(s_{3} s_{1}\right), s_{3}\right\}
$$

with the interval sequences

$$
\begin{aligned}
& \left|\left(s_{3} s_{1}\right), s_{3}\right|\left(s_{3} s_{1}\right), s_{3}\left|s_{3} /\left(s_{3} s_{1}\right)\right| s_{3} /\left(s_{3} s_{1}\right)\left|s_{3} /\left(s_{3} s_{1}\right)\right| s_{3} / s_{3} \\
& \left|\left(s_{3} s_{1}\right), s_{3}\right|\left(s_{3} s_{1}\right), s_{3} \mid s_{3} / s_{3}\left(s_{1} \mid s_{3}\right) / s_{3}\left(s_{1} \mid s_{3}\right) / s_{3}\left(s_{1} \mid s_{3}\right) / s_{3} \\
& \left(s_{3} s_{1}\right)^{5}\left(s_{3}\right)^{7}=2, \quad N=12 .
\end{aligned}
$$

(3) The Pythagorean 7-tone scale proper is obtained by choosing the two interval factors

$$
\left\{t=s_{1}^{3} s_{2}^{2}, s=s_{3}\right\},
$$




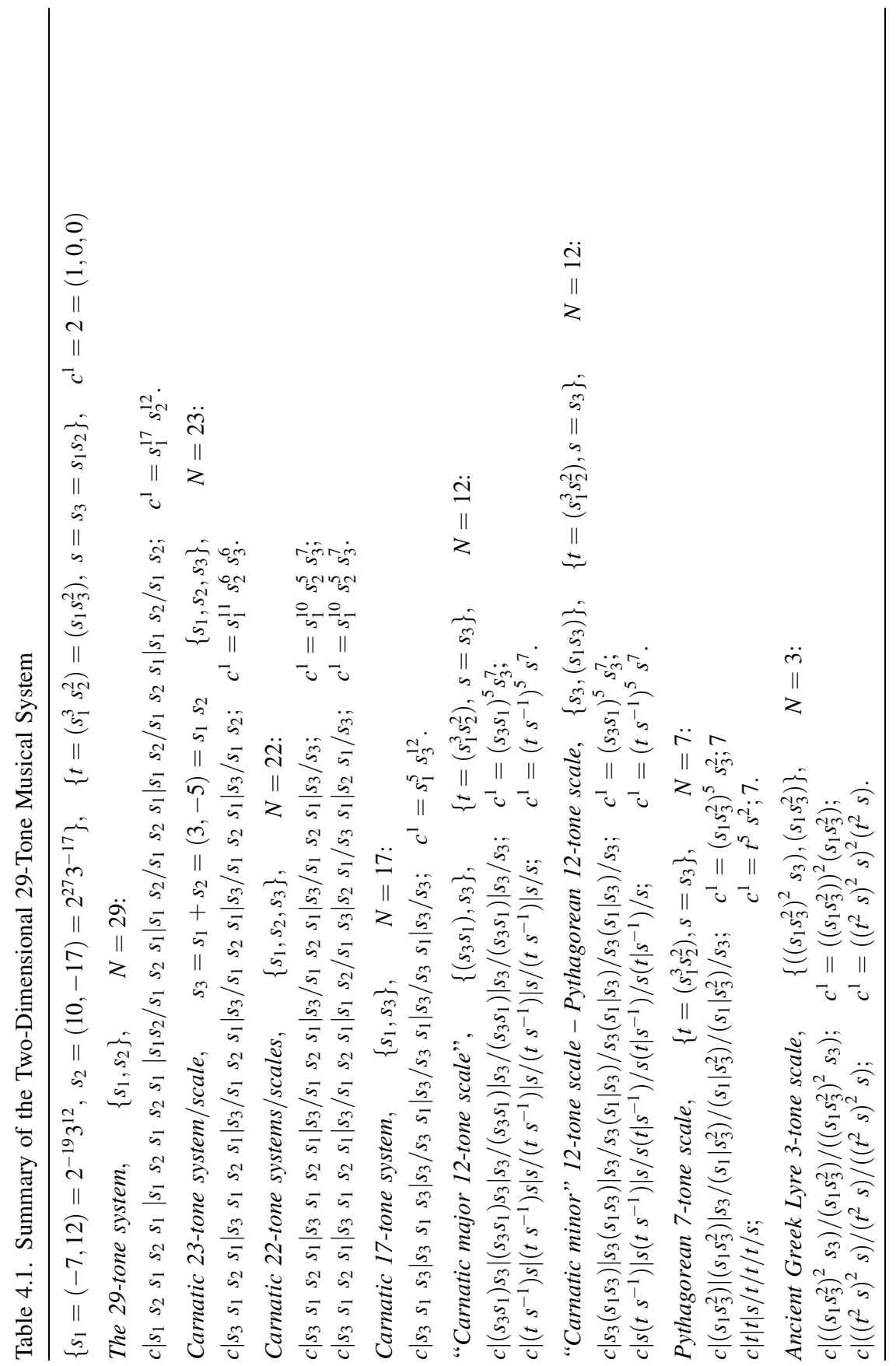


with the interval sequence

$$
|t| t \mid s / t / t / t / s, t^{5} s^{2}=\left(s_{1}^{3} s_{2}^{2}\right)^{5} s_{3}^{2}=2, \quad N=7 .
$$

(4) The two intervals for the ancient Greek Lyre are given by

$$
\left\{t^{2} s=\left(s_{1}^{7} s_{2}^{5}\right), t=\left(s_{1}^{3} s_{2}^{2}\right)\right\}, \quad t=s_{1}^{3} s_{2}^{2}, \quad s=s_{3}
$$

with

$$
\left(t^{2} s\right)^{2} t=2, \quad N=3 .
$$

The tone scale is given by the three tones

$$
c(=\bar{c}) ; t^{2} s \rightarrow \bar{f}(=f) ; t \rightarrow \bar{g}(=g) ; t^{2} s \rightarrow \bar{c}^{1}(=c) .
$$

The tones of the ancient Greek Lyre musical scale are simultaneously tones of the 3-dimensional and 2-dimensional musical systems.

Table 4.1 gives a summary of the properties of the Pythagorean plane-based tonal systems in terms of the two sruti $s_{1}$ and $s_{2}$.

Whether, or not, the hypothetical Carnatic musical scales derived in this article were ever used in practice is disputed, ref. [9]. However, the mathematical system for musical systems/scales developed in this article does, in a natural way, lead to tonal systems/scales of 29, 23, 22, 12, 7 and 3 tones, numbers which either correspond to established scales or keep coming up in discussions among scholars concerning the Carnatic scales, refs. [10], [11]. That a mathematical theory predicts precisely these numbers - and not other numbers - appears to be beyond a mere, unrelated, coincidence. In addition, in subsequent sections of this article it will be shown that the 29-tone 2-dimensional musical system developed in this article is functionally correlated to a 31-tone 3-dimensional lattice system, which in turn contains the standard chromatic musical scales as subscales. The mathematical structures and correlations discussed in this article appear to reflect themselves in theoretical discussions, as well as in practical constructions, of musical scales by musicians.

\section{Comments on the Embedding of Musical Scales and Tone Systems}

In this section the relationship between the tones of the tone systems in the 3-dimensional lattice space and the tones of the tone systems in the 2-dimensional Pythagorean sub-lattice is discussed. This is in view of embedding the 2-dimensional Pythagorean musical systems/scales into the 3-dimensional musical lattice space. 
An embedding is given if, for each tone of a 2-dimensional Pythagorean tone system, a tone in 3-dimensional lattice space can be found, such that the collection of these 3-dimensional lattice tones satisfies

(a) all the properties of a 3-dimensional lattice tone system, and

(b) the images of the 3-dimensional tones in the Pythagorean lattice space, and the images of the properties of the 3-dimensional tone system in the Pythagorean plane become the tones and the properties of the Pythagorean tone system.

The Pythagorean tone system is then said to be embedded in the 3-dimensional lattice tone system.

The Pythagorean lattice space with lattice points

$$
(n, m, 0), \quad n, m=0, \pm 1, \pm 2, \pm 3, \ldots
$$

forms a sub-lattice of the 3-dimensional lattice space with lattice points

$$
(n, m, r), \quad n, m, r=0, \pm 1, \pm 2, \pm 3, \ldots
$$

for $r=0$.

The tones

$$
\begin{array}{ccc}
c=\bar{c}=(0,0,0), & d=\bar{d}=(-1,2,0), & f=\bar{f}=(1,-1,0), \\
1 & 9 / 8 & 4 / 3 \\
g=\bar{g}=(0,1,0), & c^{1}=\bar{c}^{1}=(1,0,0), & \\
3 / 2 & 2
\end{array}
$$

are simultaneously tones of both, the 3-dimensional tone system and the Pythagorean tone system, while the tones

$$
\begin{array}{ccc}
e=(-1,1,1), & a=(0,0,1), & h(b)=(-1,2,1), \\
5 / 4 & 5 / 3 & 15 / 8
\end{array}
$$

are tones of the 3-dimensional lattice tone system, but not of the Pythagorean tone system. These tones however can be mapped onto tones of the Pythagorean-Carnatic plane by means of the projection

$$
(n, m, r)+r(-1,3,-1)=(n-r, m+3 r, 0),
$$

where the vector

$$
p=(-1,3,-1)=(2 / 1)^{-1}(3 / 2)^{3}(5 / 3)^{-1}=81 / 80
$$


has the numerical value of the syntonic comma. The 3-dimensional tones $e, a, h$, Eq. (5.4), are then related to their images $\bar{e}, \bar{a}, \bar{h}$ in the Pythagorean plane by

$$
\begin{array}{ll}
e+p=(-2,4,0)=\bar{e}, & e p=(5 / 4)(81 / 80)=81 / 64=\bar{e}, \\
a+p=(-1,3,0)=\bar{a}, & a p=(5 / 3)(81 / 80)=27 / 16=\bar{a}, \\
h+p=(-2,5,0)=\bar{h}, \quad & h p=(15 / 8)(81 / 80)=(243 / 128)=\bar{h} .
\end{array}
$$

The projection along the vector $p$ into the Pythagorean plane is however, as it was pointed out before, not one to one. The problem is then to identify, from among all the 3-dimensional lattice tones which are projected upon a given 2-dimensional tone, in a unique way that particular 3-dimensional tone which also satisfies the properties of a (possibly) larger 3-dimensional lattice tone system. In other words, a (possibly) larger 3-dimensional lattice tone system needs to be found such that the properties of the 3-dimensional lattice tone system, projected into the 2-dimensional Pythagorean lattice space, yields the Pythagorean musical system, while the properties of both systems must be simultaneously satisfied.

Such a situation arises for the case of the 29-tone 2-dimensional Pythagorean tone system. It will be shown that the 29-tone system can be embedded into the 3-dimensional lattice tone space, such that 313 -dimensional lattice tones can be determined which form a 3-dimensional musical lattice tone system. Of the 31 3-dimensional lattice tones, 29 are projected onto 29 2-dimensional lattice tones of the basic $n=0$ octave. The images of the remaining 2 tones, namely the tones $\bar{y}_{1}$ and $\bar{w}_{1}$, are in the $n=-1$ and the $n=1$ octaves of the 2-dimensional lattice tone system and are thus "lost tones". That is, these two tones are not tones of the 29-tone tone system. Also a reversal of tone sequence occurs, see Tables 6.2 and 6.3.

Another example for this relationship of mapping and embedding is the map of the hypothetical 3-dimensional 23-tone Carnatic system/scale into the Pythagorean plane resulting into the 12-tone Pythagorean musical scale. The 12-tone Pythagorean scale is, vice versa, embedded into the larger 23-tone 3-dimensional system such that, except for the tone $c$, two 3-dimensional Carnatic tones correspond to one 2-dimensional Pythagorean tone and the properties of both systems are simultaneously satisfied. This then implies a 
well defined relationship for the intervals of the two tonal systems. See Sect. 10 and ref. [10].

\section{The Three-Dimensional 31-Tone Musical Tone System}

It was shown in [1] that the interval factors for the standard chromatic tone systems can be expressed in terms of the three constant vectors (intervals)

$$
\begin{aligned}
T_{2} & =S_{2}=(1,1,-2)=27 / 25=1.08000000, \\
T_{1} & =S_{3}=(-2,1,2)=25 / 24=1.04166667, \\
S & =(2,-2,-1)=(1,1,-2)-(-1,3,-1)=S_{2} p^{-1} \\
& =16 / 15=1.06666667 .
\end{aligned}
$$

The symbols $T_{1}$ and $T_{2}$ denote the two (distinct) tones of the 3 -dimensional musical system, while the symbol $S$ denotes its semitone. The symbols $S_{2}$ and $S_{3}$ have been introduced for reasons to become clear later on.

It was pointed out in the previous section that the two tones $c$ and $d$ of the chromatic musical scale are also tones of the Pythagorean musical scale, $c=\bar{c}, d=\bar{d}$. That is, these two tones are lattice points of the Pythagorean plane and satisfy the properties required by both, the chromatic tone scale and the Pythagorean tone scale,

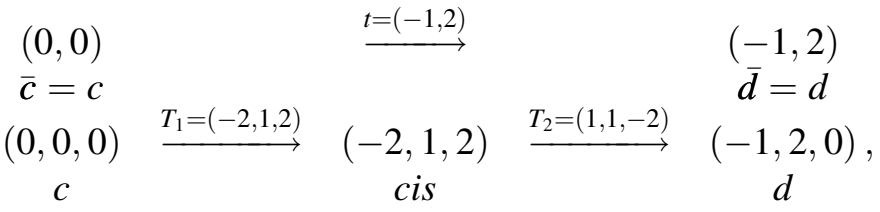

where $t=(-1,2)=9 / 8$ denotes an interval factor of the Pythagorean musical scale.

The question then arises whether additional intervals can be introduced which give rise to tones lying in between the tones $c$ and $d$ (and thus also in between the other tones of the chromatic scale) such that (a) an "enlarged chromatic system/scale" can be constructed, and (b) the projection of the "enlarged chromatic system", along the Pythagorean vector $p=(-1,3,-1)$ into the Pythagorean plane, results into the 29-tone 2-dimensional Pythagorean musical system. The 31-tone 3-dimensional system/scale will then contain all the standard chromatic scales and subscales, and by means of projection also the 29-tone 2-dimensional system and its subsystems. This will be demonstrated in what follows. 


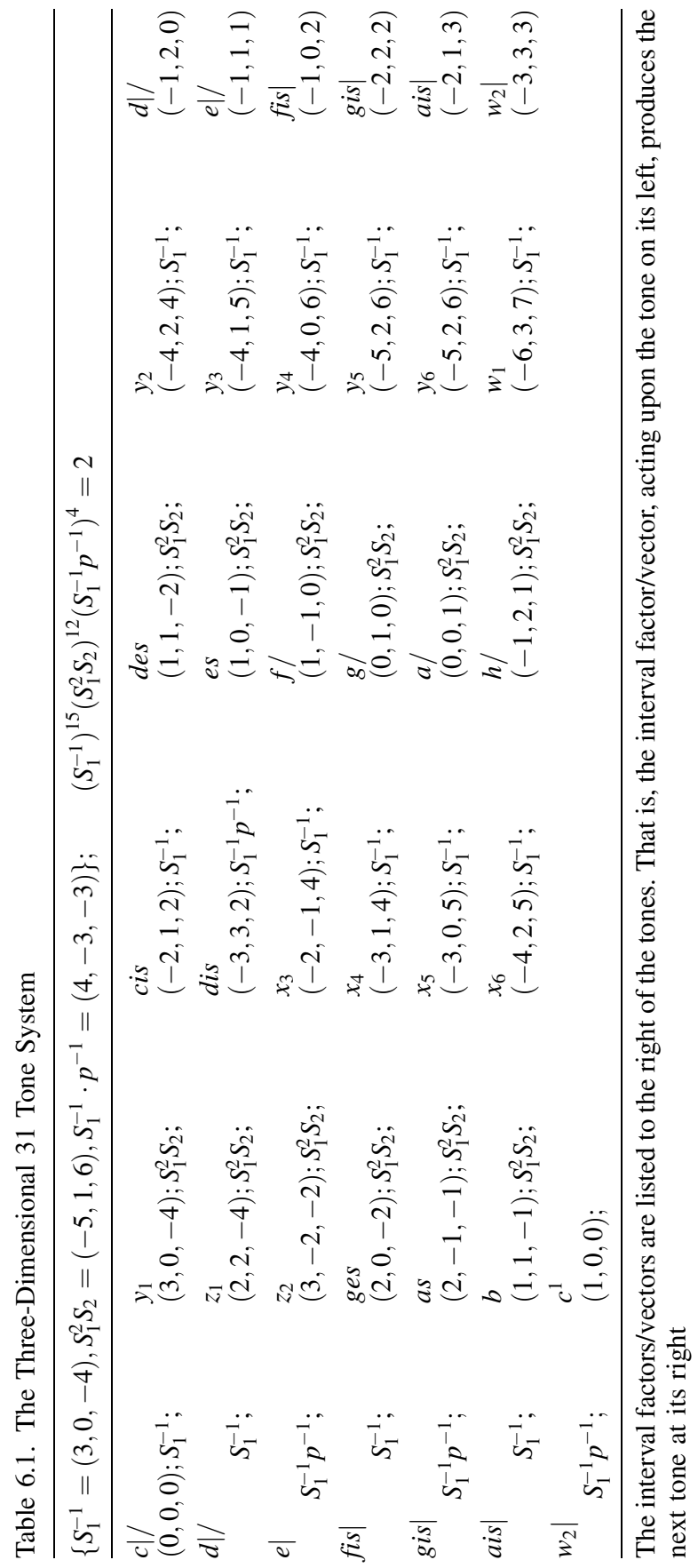




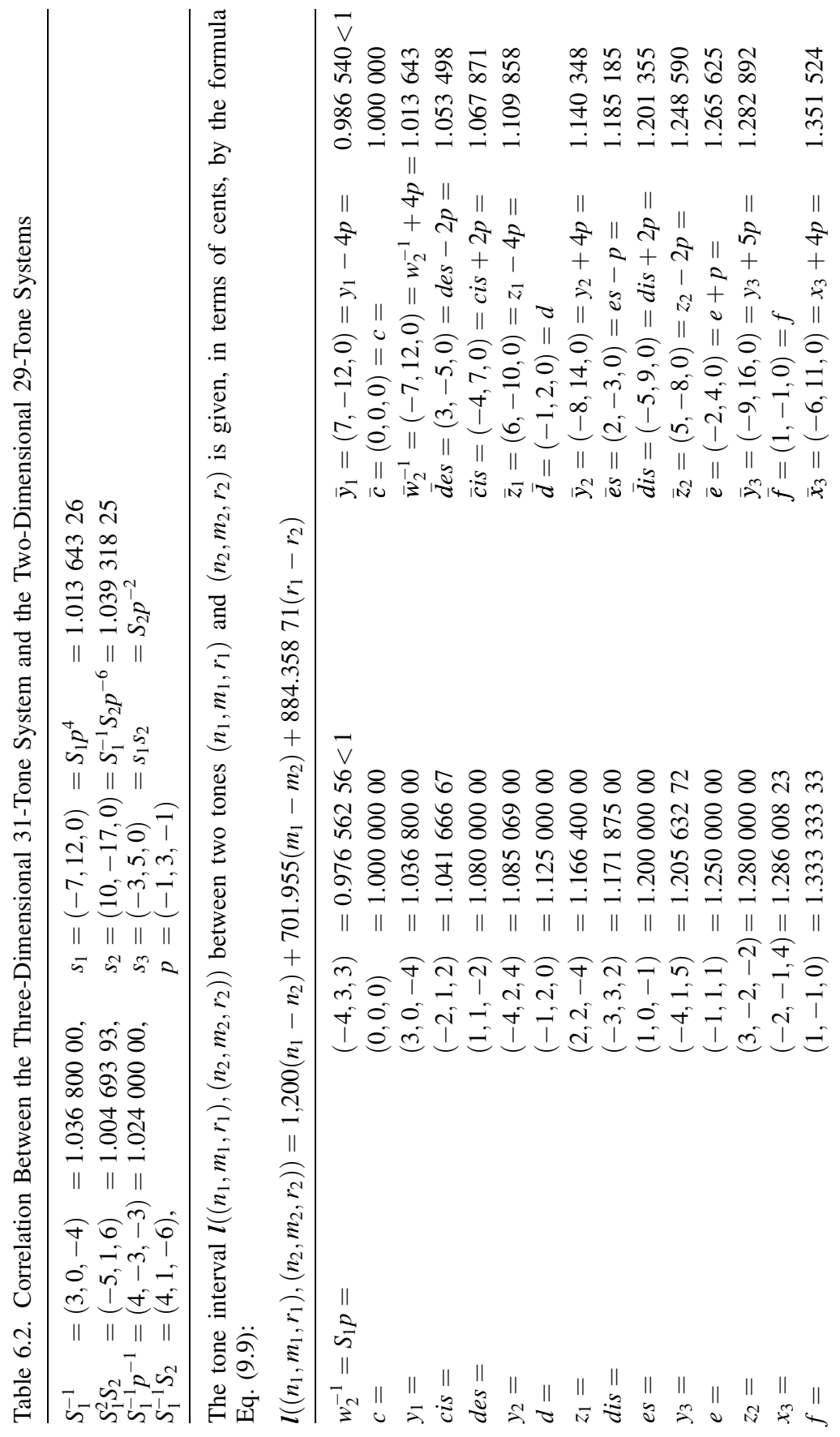




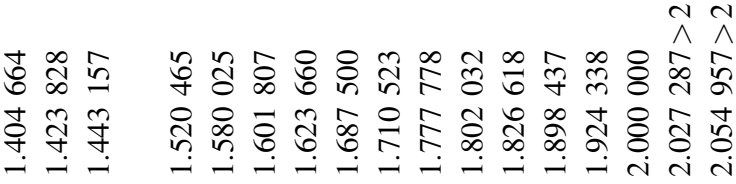

$\begin{array}{lllllllllll}\| & \| & \| & \| & \| & \| & \| & \| & \| & \| & \|\end{array}$

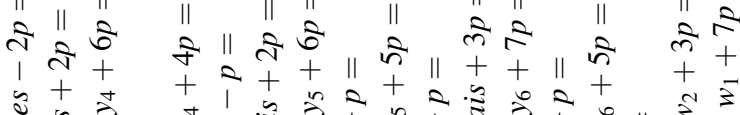
乡ँ

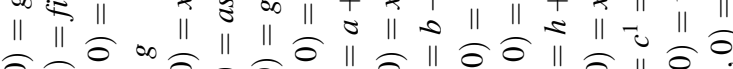
oิ 0 o o 0 món IIIEI

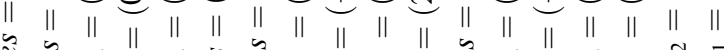

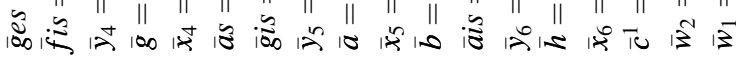

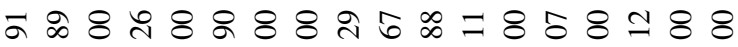

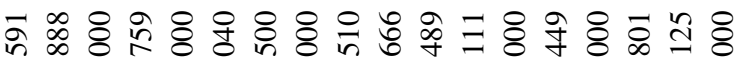
ले $\infty$ 워 - - - - - - - i-i-i-i-i ||

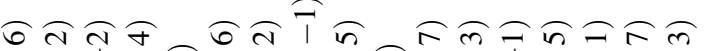
$001=0$ तथ i $=0$ m

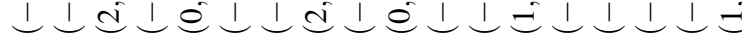

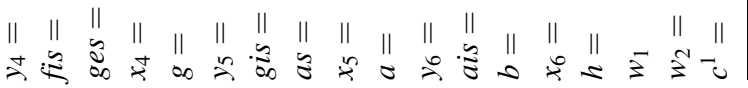


Table 6.3. Relationship Between the 31-Tone Sequence in Three-Dimensional Lattice Space and the 29-Tone Sequence in the Two-Dimensional Pythagorean Lattice Space

The sequence of the 31-tone is from left to right, as is indicated by the arrows. The images in the Pythagorean plane of the tones of the 31-tone sequence are indicated by adding a bar to the tone. The tone sequence of the images is also indicated by arrows, however there is some backtracking involved. A line of dots connects to tones in the preceding line, a line of bars connects to tones in the following line.

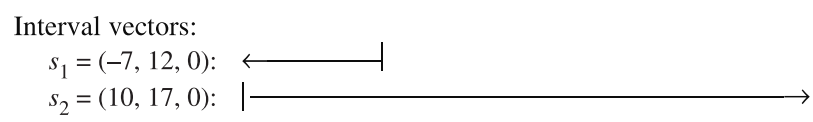

The 29 tone sequence:

$$
\left|s_{1} s_{2} s_{1} s_{2} s_{1}\right| s_{1} s_{2} s_{1} s_{2} s_{1}\left|s_{1} s_{2} s_{1} s_{2} s_{1}\right| s_{1} s_{2} s_{1} s_{2} s_{1}\left|s_{1} s_{2} s_{1} s_{2} s_{1}\right| s_{1} s_{2} s_{1} s_{2}
$$

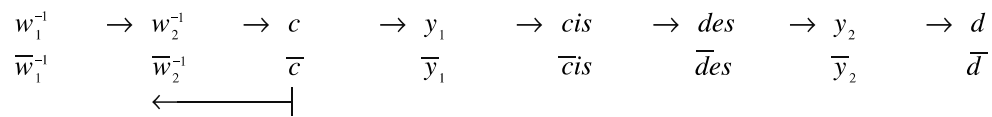
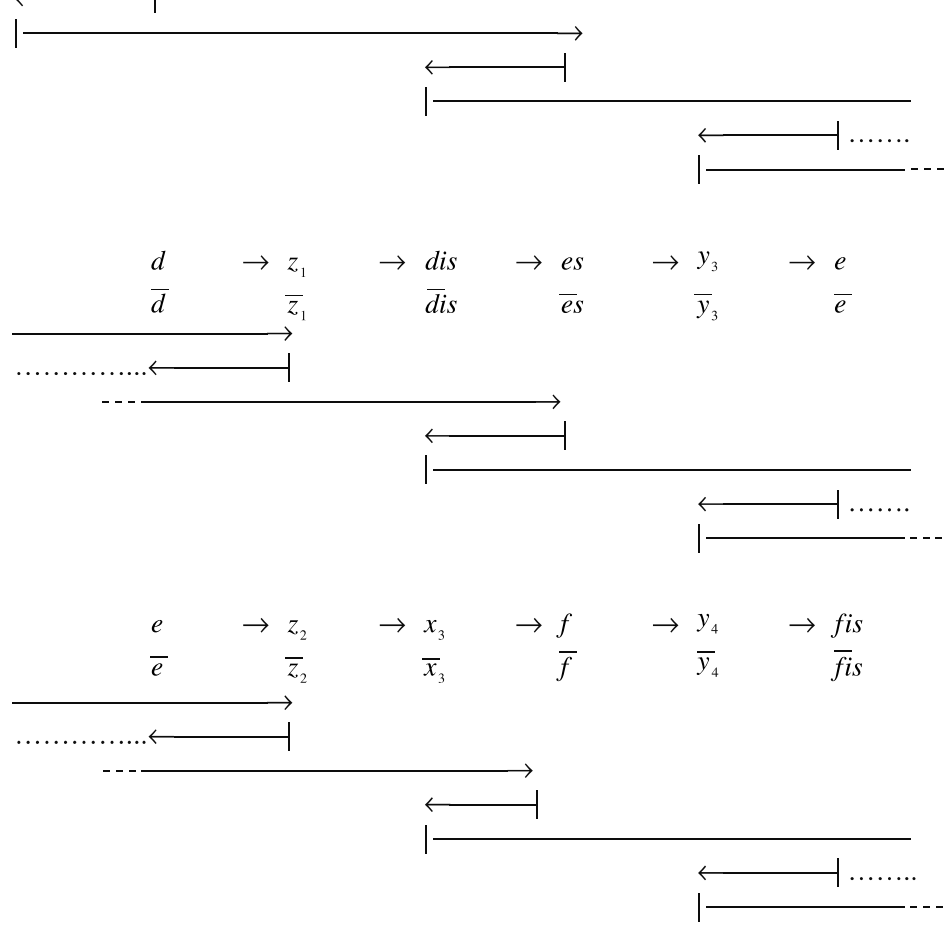
Table 6.3 (continued)
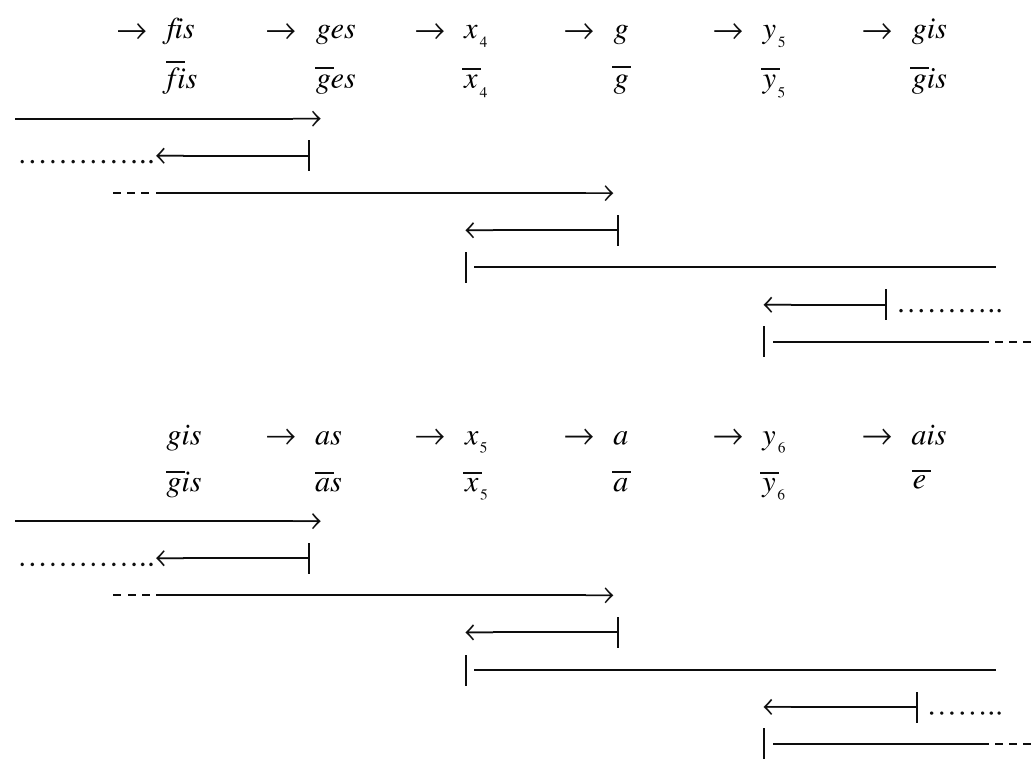

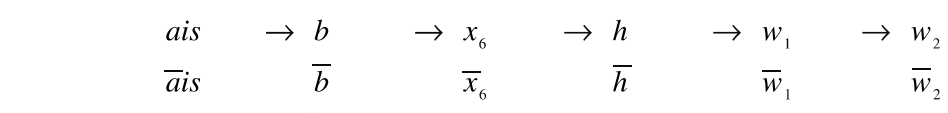
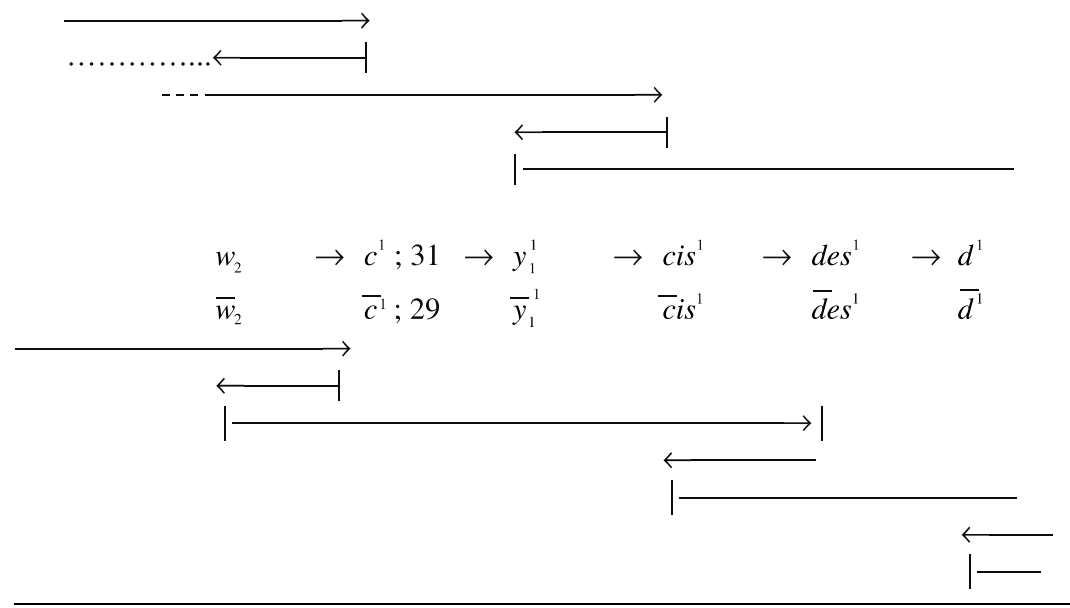
Defining the two independent vectors,

$$
\begin{gathered}
S_{1}^{-1}=s_{1}^{-1} p^{4}=(3,0,-4)=2^{3} 3^{4} 5^{-4}=648 / 625=1.03680000, \\
S_{2}=s_{1} s_{2} p^{2}=(1,1,-2)=2^{0} 3^{3} 5^{-2}=27 / 25=1.08000000
\end{gathered}
$$

and the vector

$$
\begin{aligned}
& S_{3}=s_{1}^{2} s_{2} p^{-2}=(-2,1,2)=2^{-3} 3^{-1} 5^{2}=25 / 24=1.04166667, \\
& S_{3}=S_{1} S_{2}, \quad s_{3}=s_{1} s_{2},
\end{aligned}
$$

the desired result is obtained.

The interval vectors (factors) for the 31-tone 3-dimensional music scale are obtained in terms of the constant vectors given by Eq. (6.3) as

$$
\left\{S_{1}^{-1}=(3,0,-4), S_{1}^{2} S_{2}=(-5,1,6), S_{1}^{-1} p^{-1}=(4,-3,-3)\right\}
$$

with

$$
\left(S_{1}^{-1}\right)^{15}\left(S_{1}^{2} S_{2}\right)^{12}\left(S_{1}^{-1} p^{-1}\right)^{4}=2, \quad N=31 .
$$

Thus, while the 2-dimensional musical system is defined by means of two interval vectors $s_{1}$ and $s_{2}$, the 3-dimensional (and thus the chromatic) musical system require three interval vectors, Eq. (6.4). These three interval vectors can be expressed in terms of the two sruti $s_{1}, s_{2}$, and the Pythagorean vector $p=(-1,3,-1)$ in the following way,

$$
\begin{aligned}
& S_{1}^{-1}=s_{1}^{-1} p^{4}=(3,0,-4) \quad=2^{3} 3^{4} 5^{-4} \quad=648 / 625 \\
& =1.03680000 \text {, } \\
& S_{1}^{2} S_{2}=s_{1}^{3} s_{2} p^{-6}=(-5,1,6)=2^{-6} 3^{-5} 5^{6}=15,625 / 15,552 \\
& =1.00469393 \text {, } \\
& S_{1}^{-1} p^{-1}=s_{1}^{-1} p^{3}=(4,-3,-3) \quad=2^{7} 5^{-3} \quad=128 / 125 \\
& =1.02400000 \text {. }
\end{aligned}
$$

Conversely, the two sruti $s_{1}$ and $s_{2}$ can be expressed in terms of $S_{1}^{-1}$, $S_{2}$ and $p$ as

$$
\begin{array}{llll}
s_{1}=S_{1} p^{4} & =(-7,12,0) & =2^{-19} 3^{12} & =1.01364326 \\
s_{2}=S_{1}^{-1} S_{2} p^{-6} & =(10,-17,0) & =2^{27} 3^{-17} & =1.03931825 \\
s_{3}=s_{1} s_{2}=S_{2} p^{-2} & =(3,-5,0) & =2^{8} 3^{-5} & =1.05349794
\end{array}
$$

Tables 6.1 to 6.3 list the results obtained for the 31-tone 3-dimensional tonal system. 


\section{Subsystems and Subscales of the Three-Dimensional 31-Tone System}

In this section tonal subsystems/scales of the 31-tone system are discussed. These subsystems are obtained, like it for the Pythagorean case, by combining smaller basic intervals to form new, larger intervals, in such a manner that the sequence of new intervals exhibits regularity and closes, i.e., forms a "cycle".

The set of four combined intervals (factors)

$$
\left\{S_{3}, S_{1}^{-1},\left(S_{1}^{-1} p^{-1}\right),\left(S_{2} p^{-1}\right)\right\}
$$

gives rise to the 17-tone subsystem which consists of the combined tones of the 7-tone natural diatonic musical scale, together with the 5 sharps and the 5 flats. The set of three intervals

$$
\left\{S_{3}, S_{2},\left(S_{2} p^{-1}\right)\right\}
$$

yields both the chromatic major and the chromatic minor musical scales. Which of the two scales is obtained depends upon the order of the sequence of these intervals. The set of three intervals

$$
\left\{\left(S_{1} S_{2}^{2}\right),\left(S_{1} S_{2}^{2} p^{-1}\right),\left(S_{2} p^{-1}\right)\right\}
$$

yields the natural diatonic musical scale. The set of two intervals

$$
\left\{\left(S_{1}^{2} S_{2}^{5} p^{-2}\right),\left(S_{1} S_{2}^{2}\right)\right\}
$$

yields the tonal system of the ancient Greek Lyre. Still other tonal subsystems are contained as subsets of tones of the scales discussed above.

The properties of the various interval units of the subscales are

$$
\begin{array}{lll}
S_{3} & =(-2,1,2) & =25 / 24 \\
S_{2} & =(1,1,-2) & =27 / 25 \\
\left(S_{1}^{-1} p^{-1}\right) & =(4,-3,-3) & =128 / 125, \\
\left(S_{2} p^{-1}\right) & =(2,-2,-1) & =16 / 15 \\
\left(S_{1} S_{2}^{2}\right) & =(-1,2,0) & =9 / 8 \\
\left(S_{1} S_{2}^{2} p^{-1}\right) & =(0,-1,1) & =10 / 9 \\
\left(S_{1}^{2} S_{2}^{5} p^{-2}\right) & =(1,-1,0) & =4 / 3
\end{array}
$$




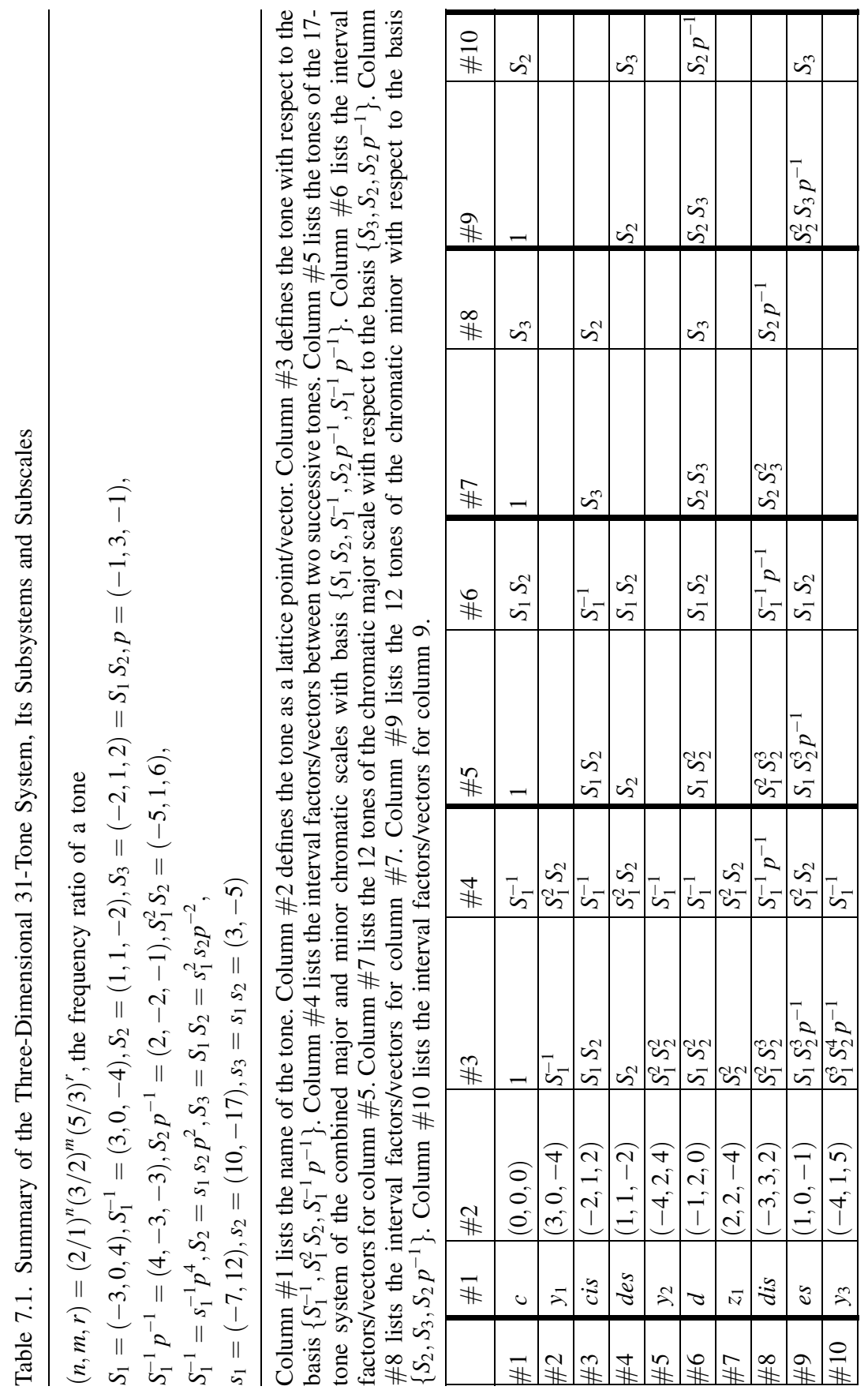


Mathematical-Physical Properties of Musical Tone Systems II

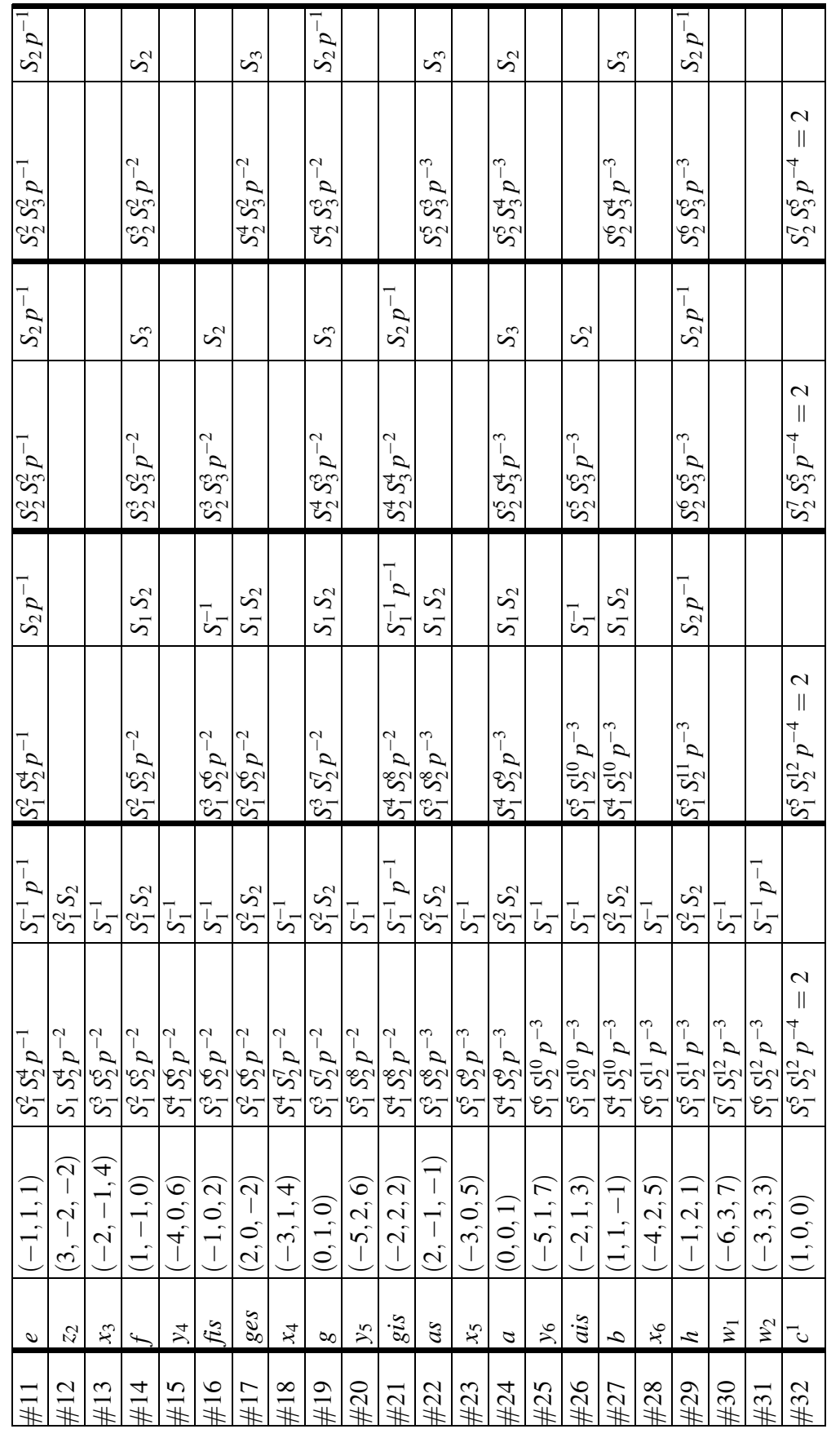




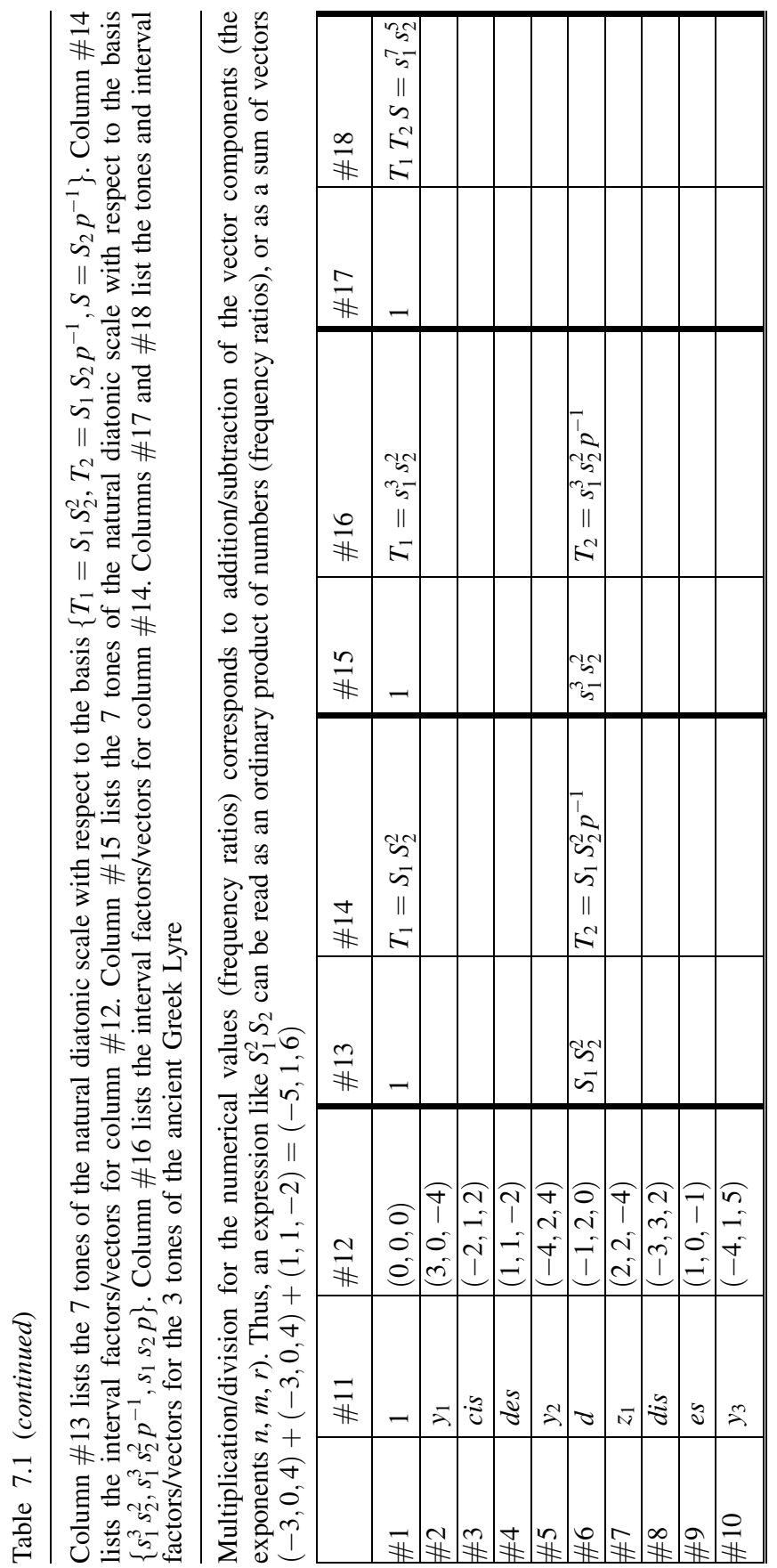




\begin{tabular}{|c|c|c|c|c|c|c|c|c|c|c|c|c|c|c|c|c|c|c|c|c|c|}
\hline & & & 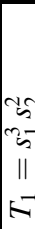 & & & & & 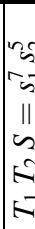 & & & & & & & & & & & & & {$\left[\begin{array}{l}N \\
\| \\
N \\
\approx \\
\approx \\
N \\
N \\
N\end{array}\right.$} \\
\hline & & & {$\left[\begin{array}{l}n \\
\text { in } \\
\text { in }\end{array}\right.$} & & & & & {$\left[\begin{array}{l}i_{5} \\
\theta_{5}\end{array}\right.$} & & & & & & & & & & & & & 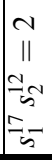 \\
\hline $\begin{array}{l}\hat{2} \\
\tilde{n} \\
\overline{5} \\
\| \\
\tilde{n}\end{array}$ & & & $\begin{array}{l}y_{2} \\
2 \\
11 \\
11 \\
-1\end{array}$ & & & & & 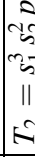 & & & & & $\begin{array}{l}25 \\
25 \\
25 \\
11 \\
-2 \\
-2\end{array}$ & & & & & $\left|\begin{array}{c}2 \\
\tilde{n} \\
\vec{n} \\
11 \\
u \\
\omega\end{array}\right|$ & & & \\
\hline 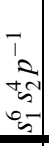 & & & $\begin{array}{l}\text { ins } \\
\text { in }\end{array}$ & & & & & {$\left[\begin{array}{l}E_{5} \\
e_{0}\end{array}\right.$} & & & & & $\begin{array}{l}1 \\
1 \\
5 \\
5 \\
n \\
n \\
5\end{array}$ & & & & & 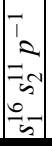 & & & 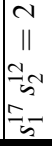 \\
\hline $\begin{array}{c}T \\
1 \\
\sim \\
\| \\
\| \\
\sim\end{array}$ & & & $\begin{array}{l}\tilde{\omega} \\
\omega \\
11 \\
-1\end{array}$ & & & & & $\begin{array}{l}\text { है } \\
\text { की } \\
11 \\
0\end{array}$ & & & & & $\begin{array}{l}\tilde{\omega} \\
\omega \\
\omega \\
\| \\
-1\end{array}$ & & & & & $\left|\begin{array}{c}1 \\
2 \\
2 \\
w^{2} \\
\| \\
\omega\end{array}\right|$ & & & \\
\hline 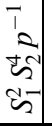 & & & $\mid \begin{array}{l}1 \\
1 \\
\text { in } \\
\text { 'ह } \\
\text { है }\end{array}$ & & & & & is & & & & & $\begin{array}{l}m \\
1 \\
2 \\
\text { हn } \\
\vdots\end{array}$ & & & & & 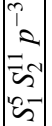 & & & 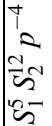 \\
\hline $\begin{array}{l}= \\
= \\
-1 \\
I\end{array}$ & $\begin{array}{c}\widehat{\imath} \\
i \\
i \\
i \\
\tilde{c}\end{array}$ & $\begin{array}{l}\vec{\partial} \\
-1 \\
i \\
i \\
i\end{array}$ & $\begin{array}{l}0 \\
- \\
-\end{array}$ & $\begin{array}{l}0 \\
0 \\
0 \\
1 \\
1\end{array}$ & $\begin{array}{l}\text { Ô } \\
0 \\
i \\
1\end{array}$ & $\begin{array}{c}\text { T } \\
1 \\
0 \\
\text { i }\end{array}$ & 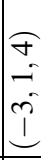 & a & $\begin{array}{l}6 \\
i \\
1 \\
1\end{array}$ & $\begin{array}{l}\overparen{2} \\
\text { i } \\
i \\
1\end{array}$ & $\begin{array}{c}7 \\
1 \\
i \\
i \\
\text { in }\end{array}$ & $\begin{array}{l}\tilde{n} \\
\tilde{0} \\
\dot{1} \\
\dot{1}\end{array}$ & $\begin{array}{c}0 \\
0 \\
0\end{array}$ & $\begin{array}{c}\approx \\
- \\
\tilde{1} \\
1\end{array}$ & $\begin{array}{c}n \\
-1 \\
i \\
1\end{array}$ & $\begin{array}{l}\approx \\
= \\
=\end{array}$ & $\begin{array}{c}0 \\
i \\
+1 \\
1\end{array}$ & $\mid \begin{array}{c}2 \\
i \\
i \\
i \\
-1\end{array}$ & $\mid \begin{array}{c}\approx \\
0 \\
0 \\
1 \\
1\end{array}$ & $\begin{array}{l}\tilde{m} \\
\dot{1} \\
\dot{1}\end{array}$ & $\begin{array}{l}0 \\
0 \\
=\end{array}$ \\
\hline 0 & $\widehat{S}$ & 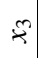 & 4 & \pm & $\stackrel{2}{2}$ & $\underset{\delta}{0}$ & $\underbrace{+}_{*}$ & or & $\stackrel{2}{2}$ & $\approx$ & 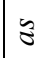 & $\ddot{\sim}$ & 0 & 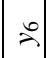 & $\cdot \tilde{\Xi}$ & 0 & 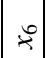 & $=$ & $\xi$ & $3^{N}$ & -0 \\
\hline$\exists$ & $\frac{\sim}{\#}$ & $\frac{m}{\#}$ & $\underset{ \pm}{ \pm}$ & $\frac{n}{\#}$ & $\frac{0}{\#}$ & 吾 & $\frac{\infty}{\#}$ & $\frac{9}{\#}$ & \# & $\overrightarrow{\mathbb{N}}$ & \# & $\underset{\#}{\#}$ & $\underset{\mathbb{Z}}{\mathbb{Z}}$ & $\stackrel{2}{\sim}$ & 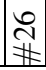 & $\underset{\#}{*}$ & \begin{tabular}{l}
$\infty$ \\
\multirow{1}{*}{} \\
$\#$
\end{tabular} & $\begin{array}{l}\stackrel{2}{A} \\
\#\end{array}$ & 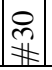 & $\ddot{m}$ & $\underset{\widetilde{N}}{\#}$ \\
\hline
\end{tabular}


Expressed in terms of the two sruti $s_{1}, s_{2}$, and the Pythagorean vector $p$, these intervals are given as

$$
\begin{aligned}
& S_{3}=\left(s_{1}^{2} s_{2} p^{-2}\right), \\
& S_{2}=\left(s_{1} s_{2} p^{2}\right), \\
& \left(S_{1}^{-1} p^{-1}\right)=\left(s_{1}^{-1} p^{3}\right), \\
& \left(S_{2} p^{-1}\right)=\left(s_{1} s_{2} p\right)=S, \\
& \left(S_{1} S_{2}^{2}\right)=\left(s_{1}^{3} s_{2}^{2}\right)=t=T_{1}, \\
& \left(S_{1} S_{2}^{2} p^{-1}\right)=\left(s_{1}^{3} s_{2}^{2} p^{-1}\right)=T_{2}, \\
& \left(S_{1}^{2} S_{2}^{5} p^{-2}\right)=\left(s_{1}^{7} s_{2}^{5}\right), \quad p^{5}=S_{2} / s_{1} s_{2}, \quad s=s_{3} . \\
& p=T_{1} / T_{2}=S / s_{1} s_{2}, \quad p^{2},
\end{aligned}
$$

For the 17-tone sub-set of the chromatic musical scales holds

$$
S_{3}^{10}\left(S_{1}^{-1}\right)^{3}\left(S_{1}^{-1} p^{-1}\right)^{2}\left(S_{2} p^{-1}\right)^{2}=(1,0,0) .
$$

For the chromatic major and minor musical scales holds

$$
S_{3}^{5} S_{2}^{3}\left(S_{2} p^{-1}\right)^{4}=(1,0,0) .
$$

For the diatonic musical scale, with the two tones $T_{1}$ and $T_{2}$, and the semitone $S$, holds

$$
\begin{array}{lll}
T_{1}=\left(S_{1} S_{2}^{2}\right) & =\left(s_{1}^{3} s_{2}^{2}\right) & =(-1,2,0), \\
T_{2}=\left(S_{1} S_{2}^{2} p^{-1}\right) & =\left(s_{1}^{3} s_{2}^{2} p^{-1}\right) & =(0,-1,1), \\
S=\left(S_{2} p^{-1}\right) & =\left(s_{1} s_{2} p\right) & =(2,-2,-1),
\end{array}
$$

with

$$
T_{1}^{3} T_{2}^{2} S^{2}=(1,0,0)=(2 / 1)^{1}(3 / 2)^{0}(5 / 3)^{0}=2
$$

and

$$
p=T_{1} T_{2}^{-1}=\left(S_{2} /\left(s_{1} s_{2}\right)\right)^{1 / 2}=(-1,3,-1)=81 / 80 .
$$

The last equation expresses the fact that the Pythagorean vector $p$ is the key to the relationship between the intervals of the Carnatic and the natural diatonic musical scales. Note that the factor $p^{-2}$ cancels in the expression $\left(S_{1}^{2} S_{2}^{5} p^{-2}\right)$, Eq. (7.5). This is the reason for the fact that the scale of the ancient Greek Lyre is contained in both the chromatic and the Carnatic musical systems. Table 7.1 contains a summary of these results. 
It can also be verified in Table 7.1 that the tone sequence of column \#9 maps, along the vector $p$, upon the standard 12-tone sequence in the Pythagorean plane

$$
\begin{aligned}
& (0,0),(3,-5),(-1,2),(2,-3),(-2,4),(1,-1),(4,-6),(0,1), \\
& (3,-4),(-1,3),(2,-2),(-2,5),
\end{aligned}
$$

while the sequence of tones given by the column \#7 maps upon the 12-tone 2-dimensional sequence

$$
\begin{aligned}
& (0,0),(-4,7),(-1,2),(-5,9),(-2,4),(1,-1),(-3,6),(0,1), \\
& (-4,8),(-1,3),(-5,10),(-2,5) .
\end{aligned}
$$

\section{Correlation of Tone Sequences in Two-Dimensional Pythagorean Plane with the Three-Dimensional 31-Tone Sequence}

The images of the chromatic major and minor 3-dimensional scales in the Pythagorean plane will be called "Pythagorean major" and "Pythagorean minor" musical scales. The tones of the chromatic major and minor 3-dimensional scales are, by means of the map along the Pythagorean vector $p=(-1,3,-1)=81 / 80$, uniquely correlated with the tones of the 2-dimensional "Pythagorean major" and "Pythagorean minor" scales.

As was pointed out in Sect. 1, the set of 3-dimensional lattice points

$$
(n+r, m-3 r, r), n, m=\text { fixed, } \quad r=0, \pm 1, \pm 2, \pm 3, \ldots
$$

is mapped onto the 2-dimensional Pythagorean lattice point

$$
\begin{gathered}
(n, m, 0)=(n+r, m-3 r, r)+r(-1,3,-1), \\
r=0, \pm 1, \pm 2, \pm 3, \ldots .
\end{gathered}
$$

This map is somewhat analogous to the map of the (2/1)-octave tones $c^{n}, n=0, \pm 1, \pm 2, \pm 3, \ldots$, along the vector $(1,0,0)$, upon the reference tone $\nu_{0}=c_{0}=c^{0}=(0,0,0)=1$, namely

$$
c^{n} /\left(2^{n} c_{0}\right)=1, \quad n=0, \pm 1, \pm 2, \pm 3, \ldots
$$

or equivalently

$$
(0,0,0)=(n, 0,0)-n(1,0,0), \quad n=0, \pm 1, \pm 2, \pm 3, \ldots .
$$

That is, Eq. (8.3) represents a map of the (2/1)-octave tones $c^{n}$, which can be considered to be musical tones equivalent to the reference tone $c_{0}$ (except for pitch, i.e. a scaling factor which cancels in musical 


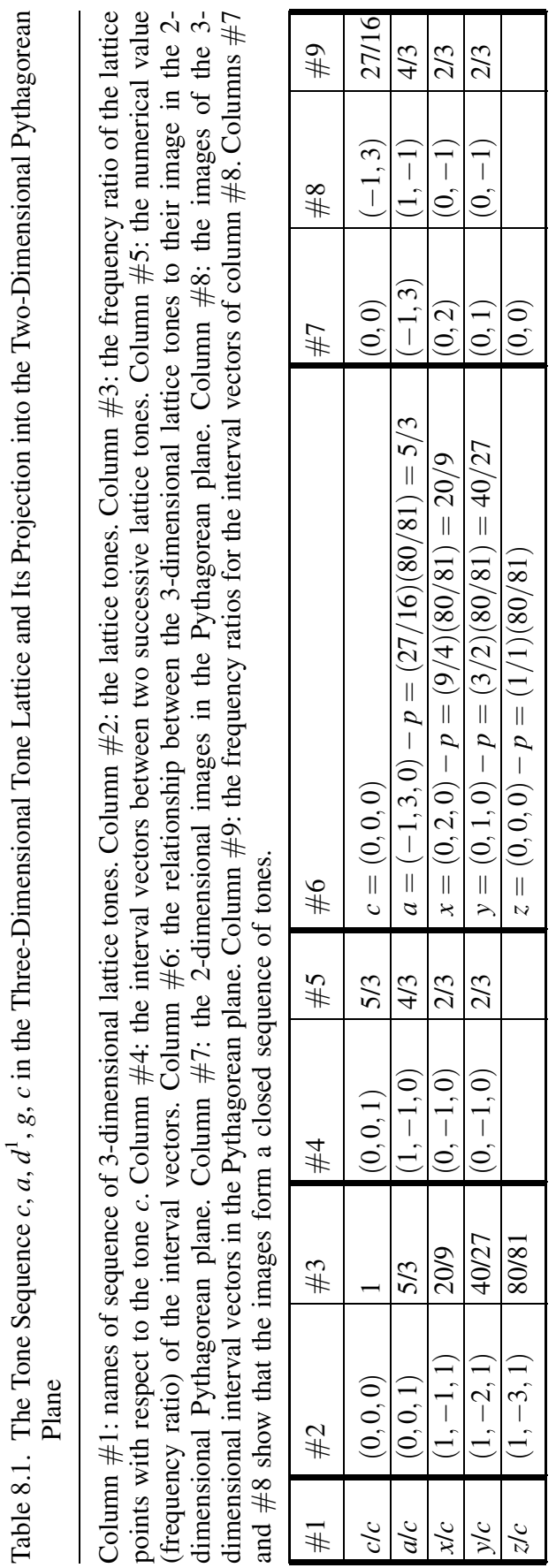


frequency ratios), upon the basic octave tone $c_{0}=c$. Thus the lattice points given by Eq. (8.1) will also be called equivalent lattice points (with respect to translations by the Pythagorean vector $p$ ).

The members of an equivalent set of lattice points are then distinguished from each other by their location on distinct Pythagorean planes labeled by $r$,

$$
(n, m, r), n, m=0, \pm 1, \pm 2, \pm 3, \ldots \text {. }
$$

The $r=0$ Pythagorean plane is the basic plane, while the other Pythagorean planes are equivalent parallel planes. These parallel planes can be considered to result from a translation, by integer multiples, by the Pythagorean vector $p=(-1,3,-1)$. Thus the musical tones on two planes, related by a translation by an integer multiple of the vector $p=(-1,3,-1)$, are equivalent musical tones (with respect to the translation $p$ ). In particular, the points equivalent to the origin $(0,0,0)=c^{0} / c_{0}=1$ of the basic Pythagorean lattice are given by the chromatic lattice points on the $r$-th Pythagorean-Carnatic plane

$$
\begin{aligned}
(0,0,0)+r(-1,3,-1) & =r(-1,3,-1), \quad r=0, \pm 1, \pm 2, \pm 3, \ldots \\
1 \times(81 / 80)^{r} & =(81 / 80)^{r}
\end{aligned}
$$

A familiar example will illustrate this point, PIERCE, ref. [12]. The frequency ratios of the tones/keys of the sequence $c, a, d^{1}, g$, $c$, with respect to the tone $c$, are

$c / c=1, \quad a / c=5 / 3, \quad d^{1} / c=9 / 4, \quad g / c=3 / 2, \quad c / c=1$.

Thus, the interval factors/vectors connecting the corresponding successive 3-dimensional lattice tones are

$$
\begin{array}{cccc}
c ; & (0,0,1) \rightarrow a ;(1,-1,0) \rightarrow x ;(0,-1,0) \rightarrow y ;(0,-1,0) \rightarrow z=p^{1} . \\
(0,0,0) \quad(0,0,1) \quad(1,-1,1) & (1,-2,1) & (1,-3,1)
\end{array}
$$

The vectors to the right of the tones are the interval vectors/factors connecting neighboring tones, while the vectors under the tones represent the tones as 3-dimensional lattice tones. It is seen that the sequence of the 3-dimensional lattice tones ends up not at the tone $c=(0,0,0)$ but at the lattice tone $p^{-1}=(1,-3,1)=80 / 81, p$ the syntonic comma.

The Pythagorean tones contained in Table 8.1 are

$$
\begin{array}{lllll}
\bar{c} & =(0,0,0) & =(0,0) & =1 & =c, \\
\bar{a} & =(-1,3,0) & =(-1,3) & =27 / 16 & =a+p, \\
\bar{d}^{1}=d^{1} & =(0,2,0) & =(0,2) & =9 / 4 & =x+p, \\
\bar{g}=g & =(0,1,0) & =(0,1) & =3 / 2 & =y+p,
\end{array}
$$




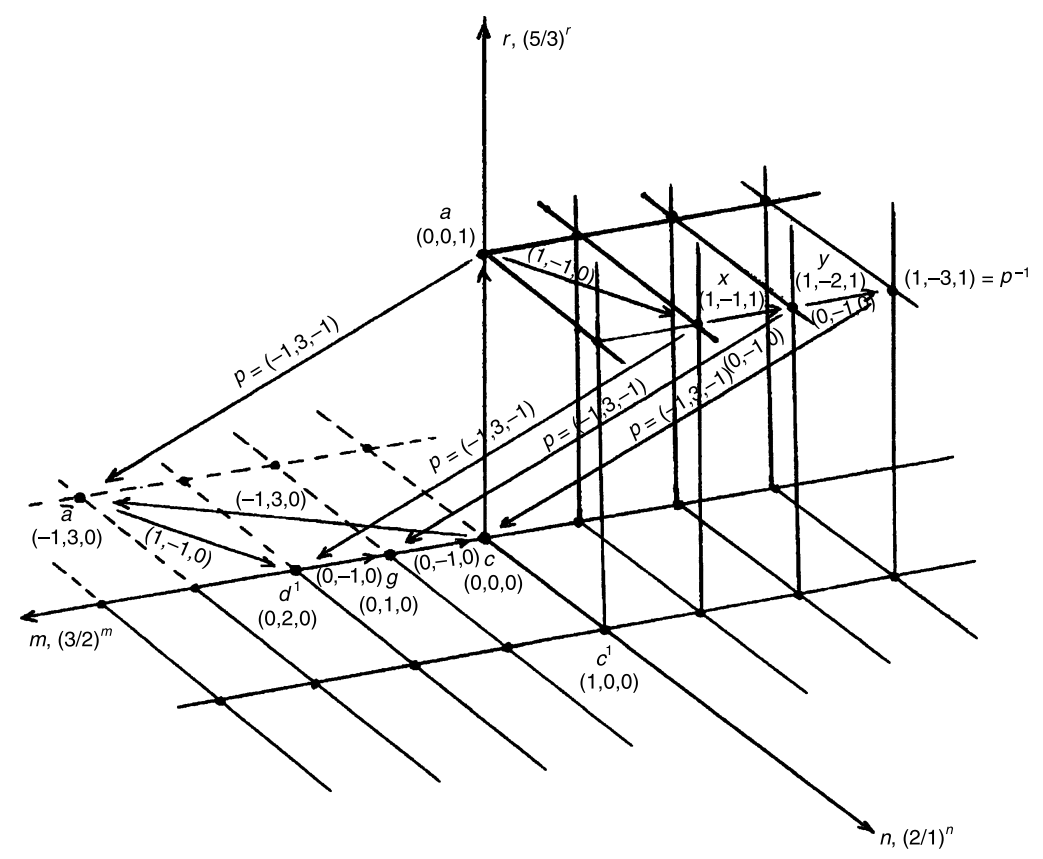

Fig. 8.1. The tone sequence $c, a, d^{1}, g, c$ in the three-dimensional tone lattice and its projection into the Pythagorean plane. This figure shows a small part of the 3-dimensional tonal lattice around the tone $(0,0,0)=1$, i.e. the tone $c$. The lattice point distances are not to scale but have been chosen such as to admit a clearer graphical representation. It is seen that the sequence of tones $c, a, d^{1}, g, c$ actually corresponds to the tone sequence $(0,0,0),(0,0,1),(1,-1,1),(1,-2,1),(1,-3,1)$, and thus leads to the tone $p^{-1}=$ $(1,-3,1)=80 / 81$, and not to the original tone $(0,0,0)=1$. The mapping, along the vector $p$, of the 31-tone 3-dimensional sequence $c, a, d^{1}, g, c$ from the chromatic lattice into the $r=0$ Pythagorean plane, yields the image sequence ("the shadow cast in the plane by the 3 -dimensional tones") $\bar{c}=c, \bar{a}, \bar{d}^{1}=d^{1}, \bar{g}=g, \bar{c}=c$ which does close. It can also be seen that the numerical values associated with these tones can be mapped onto the $(2 / 1)$-octave line $(0, \infty)$, the standard representation for the musical tones. These numerical values of the musical tones, however, do not fully represent the musical tones, but represent merely one property of the musical tones which are given as lattice

$$
\text { points }(n, m, r)
$$

with

$$
p=(-1,3,-1) \text {. }
$$

The tones $x, y, z$ are defined in the table (the reference tone $c$ is set equal to 1, except where a special point needs to be made). A graphical representation of Table 8.1 is given in Fig. 8.1.

Thus, the tone $c$ which is reached by this sequence of lattice tones is seen to be a tone $c$ which lies in the $r=1$ Pythagorean plane, a tone 
which is equivalent, with respect to the Pythagorean vector $p$, to the tone $c$ in the basic Pythagorean plane, $r=0$. The cycle of tones does not close since the tone $a=5 / 3$ leads to a tone outside the basic PythagoreanCarnatic plane. The sequence of images $\bar{c}=c, \bar{a}, \bar{d}^{1}, \bar{g}=g, \bar{c}=c$, however, closes in the Pythagorean plane. Thus the "inner structure" of the musical tones $(n, m, r)$, which characterizes the musical tones in terms of a "three octave system", the standard (2/1)-octave system and two rescaled octave systems, the (3/2)-octave system and the (5/3)octave system, provides a deeper insight into musical properties of tones, and tone systems, than their associated numerical values alone (the frequency ratios) can provide. See Fig. 8.1. (See also Figs. 8, 12 and 15 of [1]).

The correspondence between the chromatic major and minor tone scales and the "Carnatic major" and "Carnatic minor" tone scale is given by

$$
\begin{array}{llll}
\bar{c} & =(0,0,0) & =c & \\
\bar{c} i s & =(-4,7,0) & =c i s+2 p & =(-2,1,2)+2 p \\
\bar{d} e s & =(3,-5,0) & =d e s-2 p & =(1,1,-2)-2 p \\
\bar{d} & =(-1,2,0) & =d & \\
\bar{e} s & =(2,-3,0) & =e s-p & =(1,0,-1)-p \\
\bar{d} i s & =(5,-9,0) & =d i s+2 p & =(-3,3,2)+2 p \\
\bar{e} & =(-2,4,0) & =e+p & =(-1,1,1)+p \\
\bar{f} & =(1,-1,0) & =f & \\
\bar{g} e s & =(4,-6,0) & =g e s-2 p & =(2,0,-2)-2 p \\
\bar{f} i s & =(-3,6,0) & =f i s+2 p & =(-1,0,2)+2 p \\
\bar{g} & =(0,1,0) & =g & \\
\bar{a} s=(3,-4,0) & =a s-p & =(2,-1,-1)-p \\
\bar{g} i s & =(-4,8,0) & =g i s+2 p & =(-2,2,2)+2 p \\
\bar{a} & =(-1,3,0) & =a+p & =(0,0,1)+p \\
\bar{b} & =(2,-2,0) & =b-p & =(1,1,-1)-p \\
\bar{a} i s & =(-5,10,0) & =a i s+3 p & =(-2,1,3)+3 p \\
\bar{h} & =(-2,5,0) & =h+p & =(-1,2,1)+p \\
\overline{\bar{c}^{1}} & =(1,0,0) & =c^{1} &
\end{array}
$$

The list above shows that the frequencies of the pure tones of the chromatic major and the chromatic minor musical scales are, in the corresponding tones of the Carnatic system, modified by factors $p^{r}$,

$$
p^{r}=(81 / 80)^{r}, \quad r=0, \pm 1, \pm 2, \pm 3, \ldots,
$$


where $r$ indicates the $r$-th level of the Pythagorean plane, with $r=0$ denoting the basic Pythagorean plane. That is, the $r$-th Pythagorean plane is equivalent to the basic Pythagorean-Carnatic plane, modulo the vector $p$, and is obtained from it by a translation (a shift) by the vector

$$
p^{n}=n(-1,3,-1)=(-n, 3 n,-n) .
$$

For example, the chromatic tone $c i s=25 / 24$, which corresponds to the lattice point $=(-2,1,2)$ on the $r=2$ Pythagorean plane, is translated by the vector $2 p=2(-1,3,-1)=(81 / 80)^{2}$ into the $r=0$ basic Pythagorean lattice point $\bar{c} i s=(-4,7,0)$,

$$
\begin{aligned}
\bar{c} i s & =(2 / 1)^{-4}(3 / 2)^{7}=\left((2 / 1)^{-2}(3 / 2)^{1}(5 / 3)^{2}\right)\left((2 / 1)^{-2}(3 / 2)^{6}(5 / 3)^{-2}\right) \\
& =\text { cis } p^{2} .
\end{aligned}
$$

The chromatic interval factors for the chromatic major and minor scales are

$$
\begin{gathered}
\left\{\left(S_{1} S_{2}\right), S_{2},\left(S_{2} p^{-1}\right)\right\}, \quad S_{1}=(-3,0,4), \quad S_{2}=(1,1,-2), \\
p=(-1,3,-1) .
\end{gathered}
$$

These three interval factors are projected into the basic Pythagorean plane,

$$
\left(S_{1} S_{2}\right) p^{2}=\left(s_{1}^{2} s_{2}\right), \quad S_{2} p^{-2}=\left(s_{1} s_{2}\right), \quad\left(S_{2} p^{-1}\right) p^{-1}=\left(s_{1} s_{2}\right)
$$

to become the two interval factors for the "Carnatic major" and "Carnatic minor" scales

$$
\left\{\left(s_{1}^{2} s_{2}\right),\left(s_{1} s_{2}\right)\right\}
$$

Table 8.2. The Images of the Three-Dimensional Major and Minor Chromatic Musical Scales in the Two-Dimensional Pythagorean Plane

\begin{tabular}{llll}
\hline & $s_{1}=(-7,12,0)$, & $s_{2}=(10,-17,0)$, & $s=s_{3}=s_{1} s_{2}$, \\
$\bar{c}=c ; s_{3} \rightarrow \bar{d} e s ; s_{1}$ & $\rightarrow \bar{c} i s ; s_{3}$ & $\rightarrow \bar{d}=d$ & $; t=(-1,2,0)=9 / 8$ \\
$\bar{d} ; s_{3} \rightarrow \bar{e} s ; s_{1}$ & $\rightarrow \bar{d} i s ; s_{3}$ & $\rightarrow \bar{e}$ & $; t=s_{1} s_{3}^{2}=s_{1}^{3} s_{2}^{2}$ \\
$\bar{e} ; s_{3} \rightarrow \bar{f}=f ; s_{1}$ & $\rightarrow \bar{g} e s ; s_{3}$ & $\rightarrow \bar{f} i s$ & $; t$ \\
$\bar{f} i s ; s_{3} \rightarrow \bar{g}=g ; s_{1}$ & $\rightarrow \bar{a} s ; s_{3}$ & $\rightarrow \bar{g} i s$ & $; t$ \\
$\bar{g} i s ; s_{3} \rightarrow \bar{a} ; s_{1}$ & $\rightarrow \bar{b} ; s_{3}$ & $\rightarrow \bar{a} i s$ & $; t$ \\
$\bar{a} i s ; s_{3} \rightarrow \bar{h} ; s_{3}$ & $\rightarrow \bar{c}=c^{1} ;$ & $; s^{2}$ \\
$s_{1}^{5} s_{3}^{12}=2$, & $5(-7,12,0)+12(3,-5,0)=(1,0,0)$ \\
$\left(=t^{5} s^{2}\right)$ & $(=5(-1,2,0)+2(3,-5,0))$ \\
\hline
\end{tabular}


Vice versa, the three intervals

$$
\left\{\left(s_{1}^{2} s_{2}\right) p^{-2}=\left(S_{1} S_{2}\right),\left(s_{1} s_{2}\right) p^{2},\left(s_{1} s_{2}\right) p\right\}
$$

represent an embedding of the two Carnatic intervals in 3-dimensional lattice space.

The images of the chromatic minor and the major musical scales are given in Table 8.1. Tables 8.2-8.4 illustrate the correlation of the Carnatic tone sequence with respect to the 31-tone 3-dimensional tone sequence.

The images of the chromatic major and minor musical scales in the Pythagorean plane are given by the ordered sequences:

$$
\left|\left(s_{3} s_{1}\right) s_{3}\right|\left(s_{3} s_{1}\right) s_{3}\left|s_{3}\left(s_{3} s_{1}\right)\right| s_{3}\left(s_{3} s_{1}\right)\left|s_{3}\left(s_{3} s_{1}\right)\right| s_{3} s_{3}
$$

for the "Carnatic major" scale/system, and

$$
\begin{aligned}
& \left|s_{3}\left(s_{3} s_{1}\right)\right| s_{3}\left(s_{3} s_{1}\right) \mid s_{3} s_{3}\left(s_{1} \mid s_{3}\right) s_{3}\left(s_{1} \mid s_{3}\right) s_{3}\left(s_{1} \mid s_{3}\right) s_{3}, \\
& \quad \text { containing the tone } \bar{g} e s, \\
& \left|s_{3}\left(s_{3} s_{1}\right)\right| s_{3}\left(s_{3} s_{1}\right)\left|s_{3}\left(s_{3} s_{1}\right)\right|\left(s_{3} s_{1}\right) s_{3}\left|\left(s_{3} s_{1}\right) s_{3}\right|\left(s_{3} s_{1}\right) s_{3}, \\
& \quad \text { containing the tone } \bar{f} i s,
\end{aligned}
$$

for the "Carnatic minor" scale/system. See also Table 10.1 .

\section{Linearization - the Unit Interval and the Cent}

In [1] two relationships for sound frequencies were discussed. The frequency ratios $\nu / \nu_{0}$ within the basic, $n=0,(2 / 1)$-octave interval (as well as any other (2/1)-octave interval) can be expressed in the two forms

$$
\nu / \nu_{0}=1+\delta / 2 \pi, \quad 0 \leq \delta / 2 \pi \leq 1
$$

and in the form

$$
\nu / \nu_{0}=2^{\xi / 2 \pi}, \quad 0 \leq \xi / 2 \pi \leq 1,
$$

with $\nu_{0}$ an arbitrarily chosen, but fixed, reference frequency. Both equations cover the frequency values $\nu / \nu_{0}$ of the closed interval [1,2], but obviously in a linear and in an exponential manner, respectively. Thus for a fixed parameter value $\alpha$,

$$
0<\delta / 2 \pi=\xi / 2 \pi=\alpha<1,
$$

it holds

$$
\nu / \nu_{0}=1+\alpha \neq \nu^{\prime} / \nu_{0}=2^{\alpha},
$$


Table 8.3. Relationship Between the 31-Tone Three-Dimensional Sequence and the 23/22-Tone Carnatic Scales/Systems in the Pythagorean Plane

The sequence of the micro-chromatic tones is from left to right, as is indicated by the arrows. The 2-dimensional Pythagorean images of the 31-tone, 3-dimensional system, are characterized by bars. The sequence of these tones is also indicated by arrows, however there is some backtracking involved.

Interval vectors:

$S_{3}=(3,-5,0)$
$S_{1}=(-7,12,0)$
$S_{2}=(10,-17,0)$

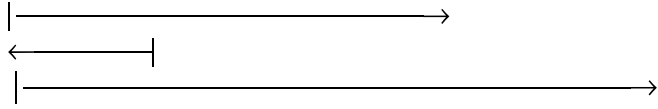

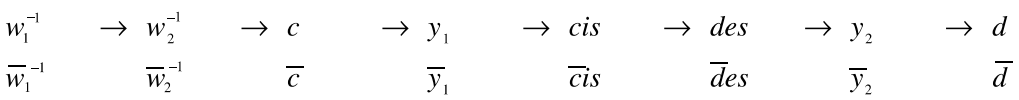
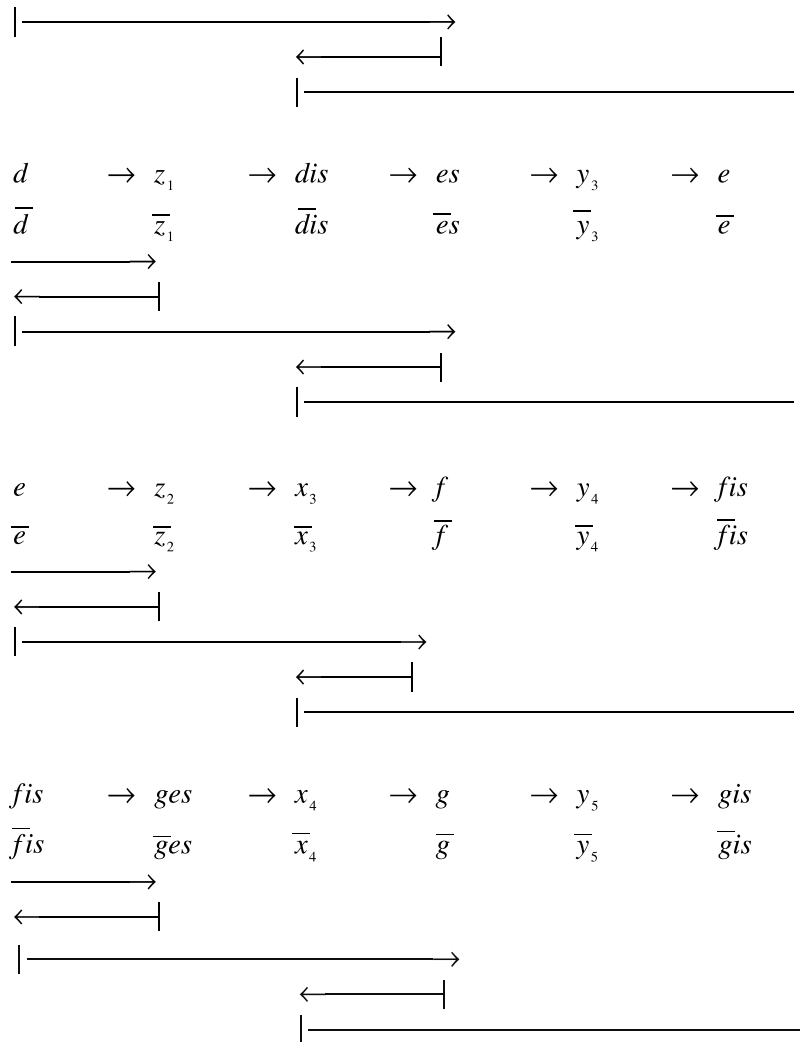
Table 8.3 (continued)
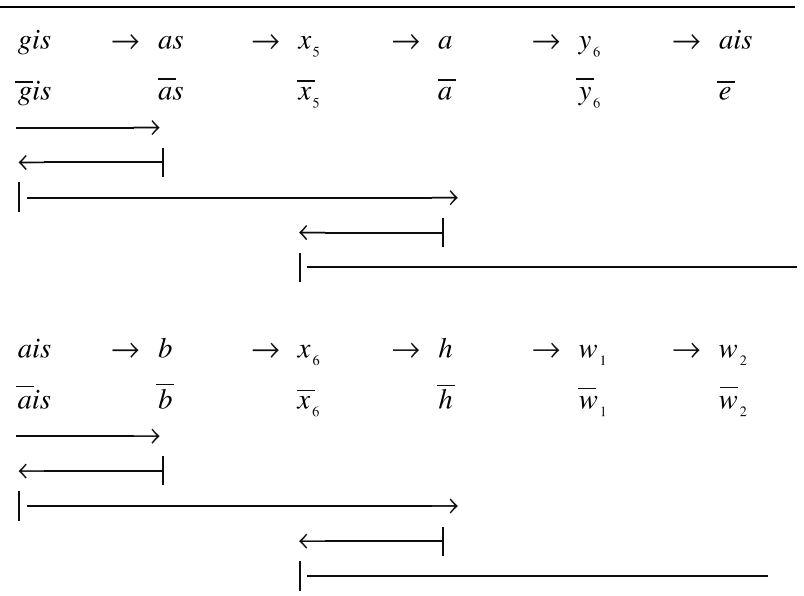

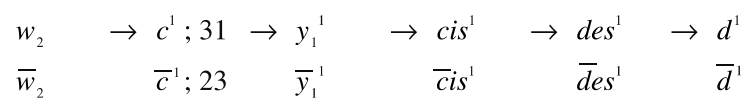

for the 23-tone Carnatic scale/tonal system, and

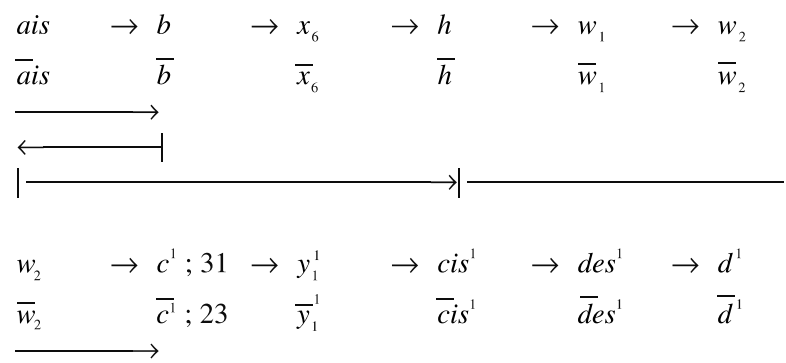

for the 22-tone Carnatic scale/tonal system.

i.e. the frequency ratios $\nu / \nu_{0}$ and $\nu^{\prime} / \nu_{0}$ are not equal. They are equal only at the two end points of the interval, namely for

$$
\alpha=\delta / 2 \pi=\xi / 2 \pi=0 \text { and } 1,
$$

with

$$
\nu / \nu_{0}=\nu^{\prime} / \nu_{0}=1 \text { and } 2 .
$$


Table 8.4. Relationship Between the 31-Tone Three-Dimensional Tonal System and the Set of 17 Tones of the Two-Dimensional "Carnatic Major" and "Carnatic Minor" Scales

The sequence of the 3-dimensional tones is from left to right, as is indicated by the arrows. The Pythagorean 2-dimensional images of the 3-dimensional tones are characterized by a bar. The sequence of the 2-dimensional tones is also indicated by arrows, however there is some backtracking involved.

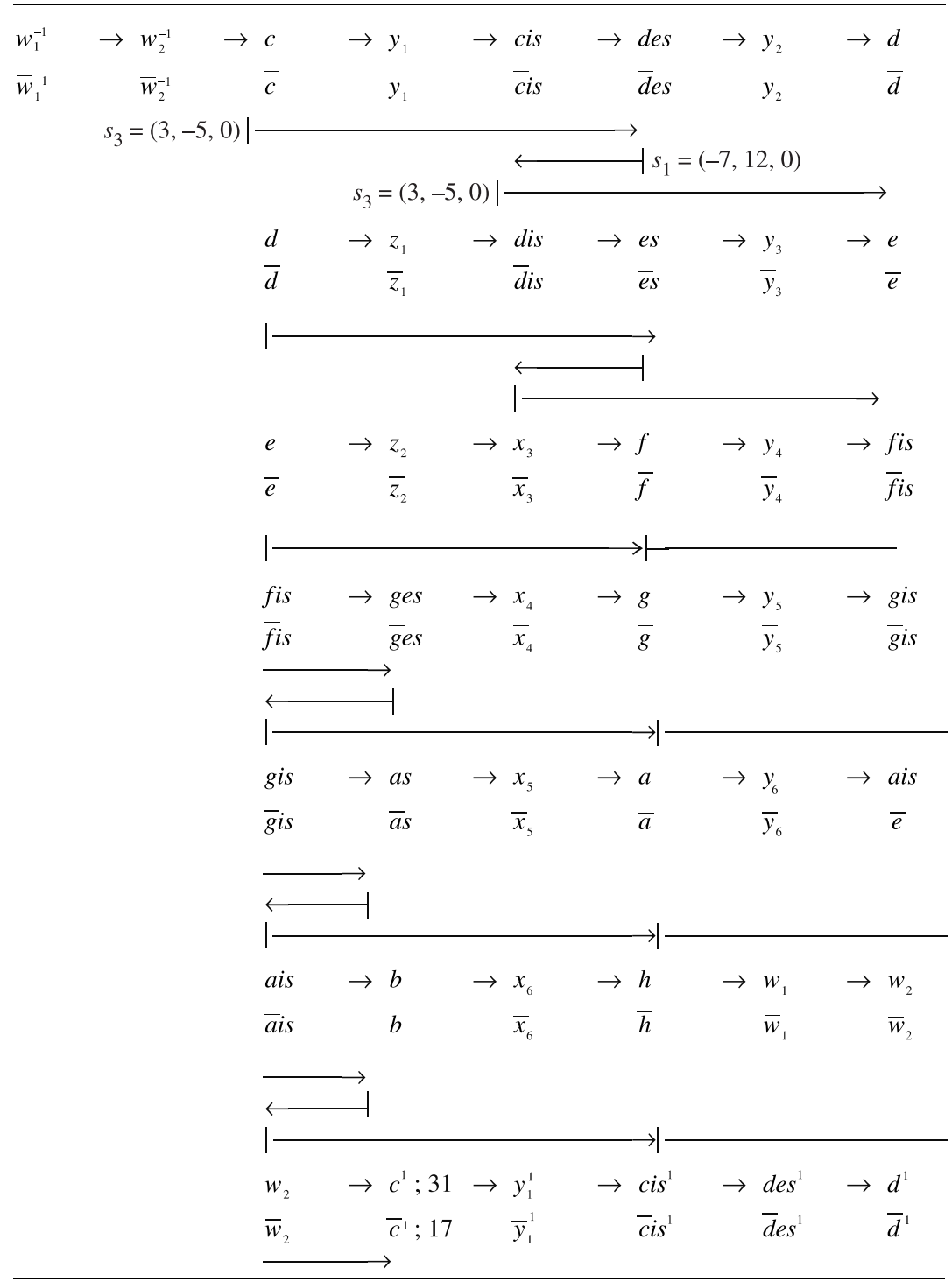


Table 8.5. Relationship Between the 31-Tone Three-Dimensional Sequence and the 7-Tone Two-Dimensional Pythagorean Sequence of Tones

The sequence of the 3-dimensional tones is from left to right, as is indicated by the arrows. The Pythagorean 2-dimensional images of the 3-dimensional 31-tone system are characterized by a bar. $\left(s_{1} s_{3}\right)=(-4,7,0), s_{3}=(3,-5,0)$

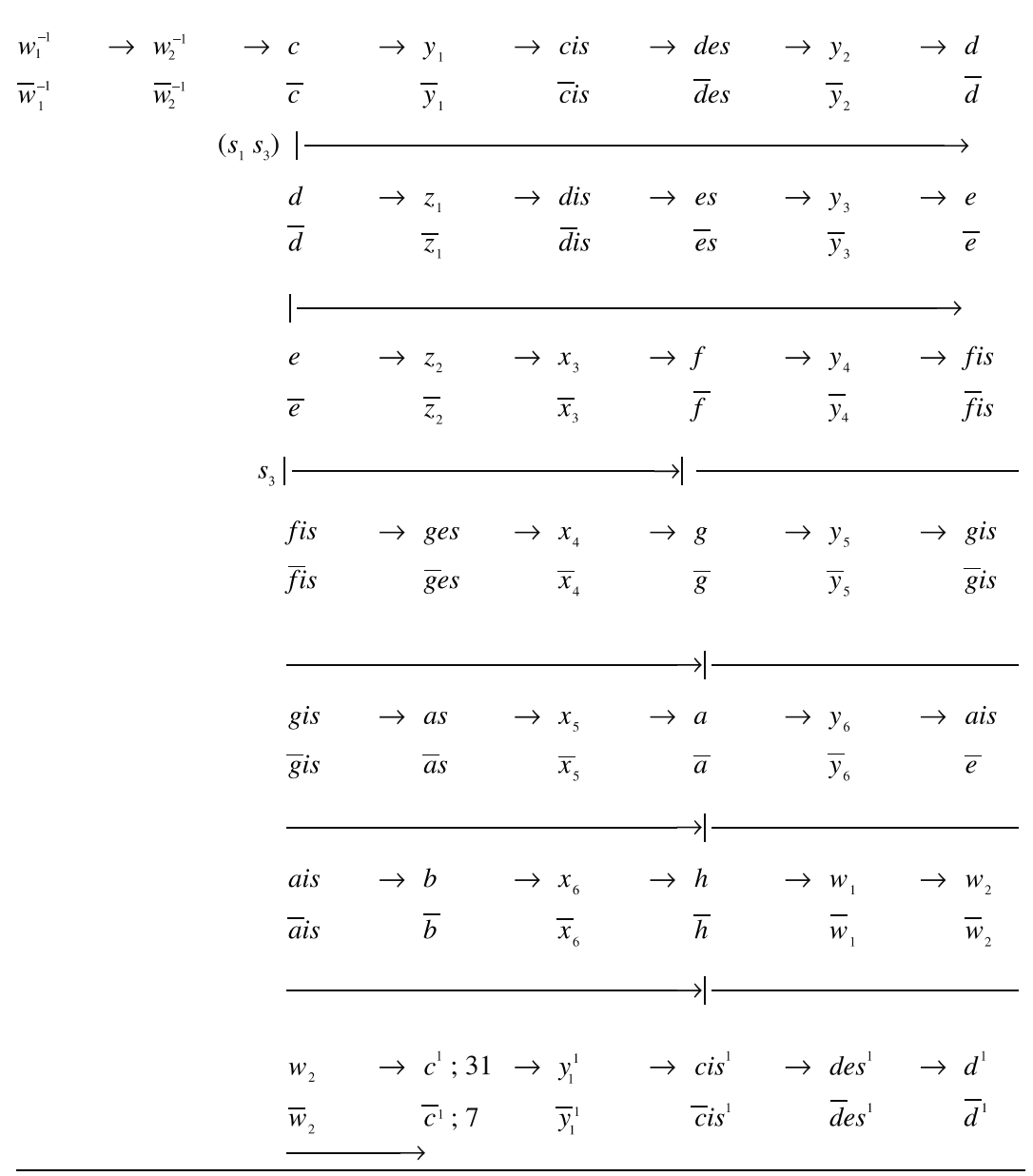

That is, the relationship between the two parameters $\delta / 2 \pi$ and $\xi / 2 \pi$ is given by the logarithmic law

$$
\xi / 2 \pi=\log _{2}(1+\delta / 2 \pi),
$$

which represents the inverse function to the exponential law, Eq. (9.2), see also refs. [3], [5]. The two functions, Eqs. (9.2) and (9.5), being 
Table 9.1. Interval Vectors, Cents, and Interval Factors for the 31-Tone ThreeDimensional Musical System

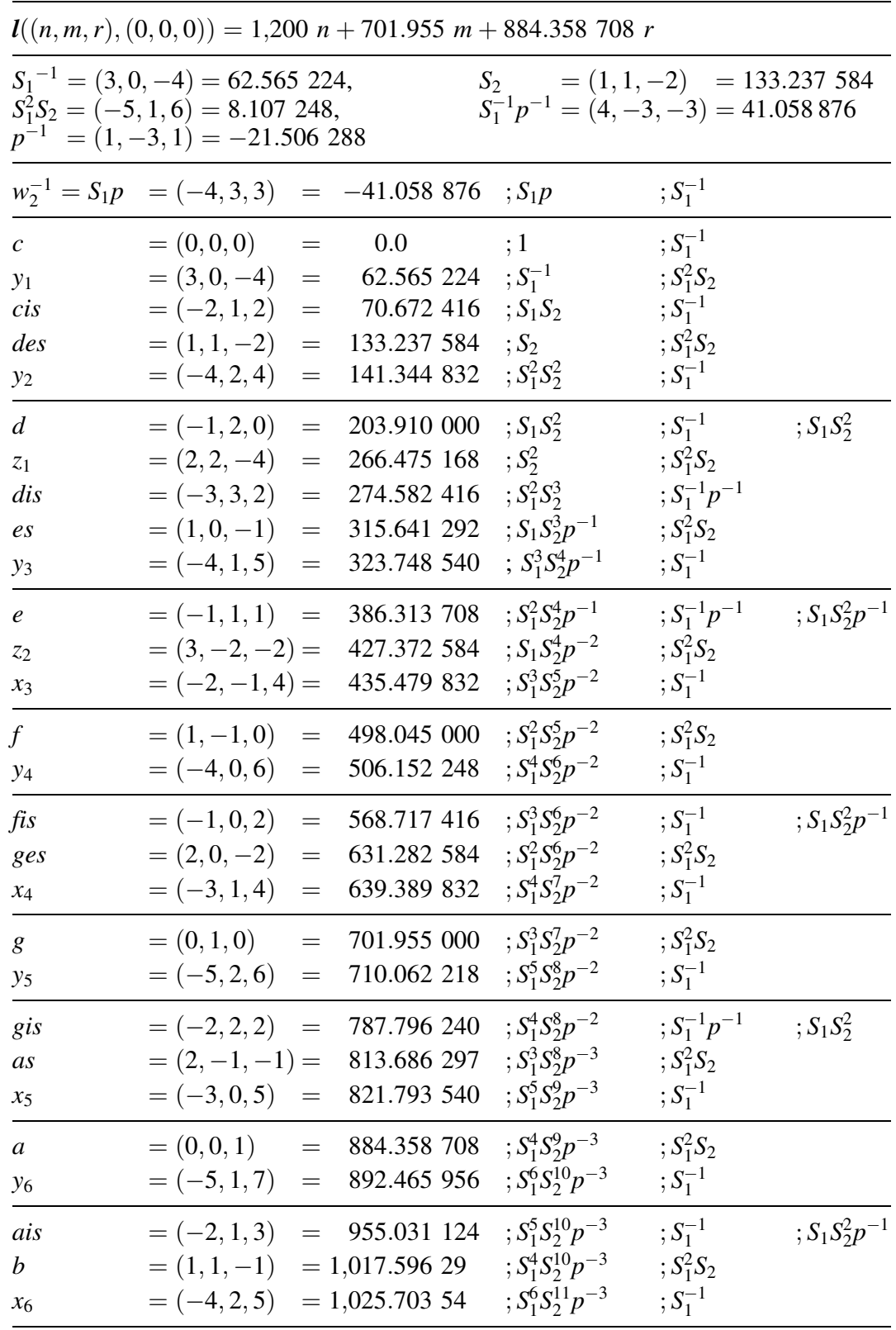


Table 9.1 (continued)

\begin{tabular}{llllll}
\hline$h$ & $=(-1,2,1)$ & $=1,088.26871$ & $; S_{1}^{5} S_{2}^{11} p^{-3}$ & $; S_{1}^{2} S_{2}$ & \\
$w_{1}=(-6,3,7)$ & $=1,096.37596$ & $; S_{1}^{7} S_{2}^{12} p^{-3}$ & $; S_{1}^{-1}$ & \\
$w_{2}=(-3,3,3)$ & $=1,158.94112$ & $; S_{1}^{6} S_{2}^{12} p^{-3}$ & $; S_{1}^{-1} p^{-1}$ & $; S_{1} S_{2}^{2}$ \\
$c^{1}=(1,0,0)$ & $=1,200.00000$ & $; S_{1}^{5} S_{2}^{12} p^{-4}=2$ & $; S_{1}^{-1} p^{-1}$ & \\
\hline$\left|\left(S_{1} S_{2}^{2}\right)\right|\left(S_{1} S_{2}^{2} p^{-1}\right)\left|\left(S_{2} p^{-1}\right)\right|\left(S_{1} S_{2}^{2}\right)\left|\left(S_{1} S_{2}^{2} p^{-1}\right)\right|\left(S_{1} S_{2}^{2}\right) \mid\left(S_{2} p^{-1}\right) ;$ & \\
$\left(S_{1} S_{2}^{2}\right)^{3}\left(S_{1} S_{2}^{2} p^{-1}\right)^{2}\left(S_{2} p^{-1}\right)^{2}=2$, the 7-tone natural diatonic scale \\
\hline
\end{tabular}

Table 9.2. Interval Vectors, Cents, and Interval Factors for the 29-Tone Two-Dimensional Musical System

\begin{tabular}{|c|c|c|c|c|}
\hline \multicolumn{5}{|c|}{$\boldsymbol{l}((n, m, 0),(0,0,0))=1,200 n+701.955 m$} \\
\hline \multicolumn{5}{|c|}{$\begin{array}{l}s_{1}=(-7,12,0)=23.46 \mathrm{cent} \\
s_{2}=(10,-17,0)=66.765 \mathrm{cent} \\
p=(-1,3,-1)=21.506288 \mathrm{cent}\end{array}$} \\
\hline $\bar{y}_{1}$ & $=(7,-12,0)$ & $=y_{1}-4 p$ & $=-23.46$ & $; s_{1}^{-1}$ \\
\hline $\begin{array}{l}\bar{c} \\
\bar{w}_{2}^{-1} \\
\bar{d}_{e s} \\
\bar{c} i s \\
\bar{z}_{1}\end{array}$ & $\begin{array}{l}=(0,0,0) \\
=(-7,12,0) \\
=(3,-5,0) \\
=(-4,7,0) \\
=(6,-10,0)\end{array}$ & $\begin{array}{l}=c \\
=w_{2}^{-1}+4 p \\
=d e s-2 p \\
=c i s+2 p \\
=z_{1}-4 p\end{array}$ & $\begin{array}{l}=\quad 0 \\
=\quad 23.46 \\
=\quad 90.225 \\
=113.685 \\
=180.45\end{array}$ & $\begin{array}{l}; 1 \\
; s_{1} \\
; s_{1} s_{2} \\
; s_{1}^{2} s_{2} \\
; s_{1}^{2} s_{2}^{2}\end{array}$ \\
\hline $\begin{array}{l}\bar{d} \\
\bar{y}_{2} \\
\bar{e} s \\
\bar{d}_{i s} \\
\bar{z}_{2}\end{array}$ & $\begin{array}{l}=(-1,2,0) \\
=(-8,14,0) \\
=(2,-3,0) \\
=(-5,9,0) \\
=(5,-8,0)\end{array}$ & $\begin{array}{l}=d \\
=y_{2}+4 p \\
=e s-p \\
=d i s+2 p \\
=z_{2}-2 p\end{array}$ & $\begin{array}{l}=203.91 \\
=227.37 \\
=294.135 \\
=317.595 \\
=384.36\end{array}$ & $\begin{array}{l}; s_{1}^{3} s_{2}^{2}=t \\
; s_{1}^{4} s_{2}^{2} \\
; s_{1}^{4} s_{2}^{3} \\
; s_{1}^{5} s_{2}^{3} \\
; s_{1}^{5} s_{2}^{4}\end{array}$ \\
\hline $\begin{array}{l}\bar{e} \\
\bar{y}_{3} \\
\bar{f} \\
\bar{x}_{3} \\
\bar{g} e s\end{array}$ & $\begin{array}{l}=(-2,4,0) \\
=(-9,16,0) \\
=(1,-1,0) \\
=(-6,11,0) \\
=(4,-6,0)\end{array}$ & $\begin{array}{l}=e+p \\
=y_{3}+5 p \\
=f \\
=x_{3}+4 p \\
=g e s-2 p\end{array}$ & $\begin{array}{l}=407.82 \\
=431.28 \\
=498.054 \\
=521.505 \\
=588.27\end{array}$ & $\begin{array}{l}; s_{1}^{6} s_{2}^{4}=t^{2} \\
; s_{1}^{7} s_{2}^{4} \\
; s_{1}^{7} s_{2}^{5} \\
; s_{1}^{8} s_{2}^{5} \\
; s_{1}^{8} s_{2}^{6}\end{array}$ \\
\hline $\begin{array}{l}\bar{f} i s \\
\bar{y}_{4} \\
\bar{g} \\
\bar{x}_{4} \\
\bar{a} s\end{array}$ & $\begin{array}{l}=(-3,6,0) \\
=(-10,18,0) \\
=(0,1,0) \\
=(-7,13,0) \\
=(3,-4,0)\end{array}$ & $\begin{array}{l}=f i s+2 p \\
=y_{4}+6 p \\
=g \\
=x_{4}+4 p \\
=a s-p\end{array}$ & $\begin{array}{l}=611.73 \\
=635.19 \\
=701.955 \\
=725.415 \\
=792.18\end{array}$ & $\begin{array}{l}; s_{1}^{9} s_{2}^{6}=t^{3} \\
; s_{1}^{10} s_{2}^{6} \\
; s_{1}^{10} s_{2}^{7} \\
; s_{1}^{11} s_{2}^{7} \\
; s_{1}^{11} s_{2}^{8}\end{array}$ \\
\hline
\end{tabular}


Table 9.2 (continued)

\begin{tabular}{|c|c|c|c|c|}
\hline $\bar{g} i s$ & $=(-4,8,0)$ & $=a s+2 p$ & $=815.64$ & $; s_{1}^{12} s_{2}^{8}=t^{4}$ \\
\hline $\bar{y}_{5}$ & $=(-11,20,0)$ & $=y_{5}+6 p$ & $=839.1$ & $; s_{1}^{13} s_{2}^{8}$ \\
\hline $\bar{a}$ & $=(-1,3,0)$ & $=a+p$ & $=905.865$ & $; s_{1}^{13} s_{2}^{9}$ \\
\hline $\bar{x}_{5}$ & $=(-8,15,0)$ & $=x_{5}+5 p$ & $=929.325$ & $; s_{1}^{14} s_{2}^{9}$ \\
\hline $\bar{b}$ & $=(2,-2,0)$ & $=b-p$ & $=996.09$ & $; s_{1}^{14} s_{2}^{10}$ \\
\hline $\bar{a} i s$ & $=(-5,10,0)$ & $=a i s+3 p$ & $=1,019.55$ & $; s_{1}^{15} s_{2}^{10}=t^{5}$ \\
\hline $\bar{y}_{6}$ & $=(-12,22,0)$ & $=y_{6}+7 p$ & $=1,043.01$ & $; s_{1}^{16} s_{2}^{10}$ \\
\hline $\bar{h}$ & $=(-2,5,0)$ & $=h+p$ & $=1,109.775$ & $; s_{1}^{16} s_{2}^{11}$ \\
\hline $\bar{x}_{6}$ & $=(-9,17,0)$ & $=x_{6}+5 p$ & $=1,133.235$ & $; s_{1}^{17} s_{2}^{11}$ \\
\hline $\bar{c}^{1}$ & $=(1,0,0)$ & $=c^{1}$ & $=1,200$ & $; s_{1}^{17} s_{2}^{12}=2$ \\
\hline $\bar{w}_{2}$ & $=(-6,12,0)$ & $=w_{2}+3 p$ & $=1,223.46$ & $; s_{1}^{18} s_{2}^{12}$ \\
\hline $\bar{w}_{1}$ & $\begin{aligned} s^{1} & =(-13,24,0) \\
t & =\left(s_{1}^{3} s_{2}^{2}\right)\end{aligned}$ & $\begin{array}{l}=w_{1}+7 p \\
/ t / t / t / t / t / t s_{1}^{-1}\end{array}$ & $=1,246.92$ & $; s_{1}^{19} s_{2}^{12}$ \\
\hline
\end{tabular}

The sequence of intervals given below represents a 22/23-tone musical system given in 2-dimensional lattice space:

$\left|\left(s_{1} s_{2}\right) s_{1} s_{2} s_{1}\right|\left(s_{1} s_{2}\right) s_{1} s_{2} s_{1}\left|\left(s_{1} s_{2}\right) s_{1} s_{2} s_{1}\right|\left(s_{1} s_{2}\right) s_{1} s_{2} s_{1}\left|s_{2} s_{1}\left(s_{1} s_{2}\right) s_{1}\right| s_{2} s_{1}\left(s_{1} s_{2}\right)$

inverse to each other, carry the same information. A physical law, represented by a mathematical function, needs to be unique. This requirement of uniqueness of a functional relationship led to the postulate that "musical lattice tones" are defined for the parameter values $\delta / 2 \pi=\xi / 2 \pi=0$ and 1 only, and that this requirement holds for each of the three octave systems based upon the frequency ratios $(2 / 1),(3 / 2)$ and $(5 / 3)$. This then led to the introduction of the 3dimensional scaled lattice space for musical tones, see also ref. [3].

The ratio of two distinct frequency ratios $\nu_{1} / \nu_{0}$ and $\nu_{2} / \nu_{0}$ (i.e. two distinct musical tones) is given by

$$
\begin{gathered}
\left(\nu_{2} / \nu_{0}\right) /\left(\nu_{1} / \nu_{0}\right)=\nu_{2} / \nu_{1}=\left(1+\delta_{2} / 2 \pi\right) /\left(1+\delta_{1} / 2 \pi\right) \\
=2^{\xi_{2} / 2 \pi} / 2^{\xi_{1} / 2 \pi}=2^{\left(\xi_{2} / 2 \pi\right)-\left(\xi_{1} / 2 \pi\right)} .
\end{gathered}
$$

Taking the logarithm with base 2 one obtains

$$
\begin{aligned}
\log _{2}\left(\nu_{2} / \nu_{1}\right) & =\log _{2}\left(1+\delta_{2} / 2 \pi\right)-\log _{2}\left(1+\delta_{1} / 2 \pi\right) \\
& =\left(\xi_{2} / 2 \pi\right)-\left(\xi_{1} / 2 \pi\right)=\boldsymbol{l} .
\end{aligned}
$$

That is, the ratio of the two frequencies $\nu_{2} / \nu_{1}$ (the ratio of the two musical tones $\left(\nu_{1} / \nu_{0}\right)$ and $\left.\left(\nu_{2} / \nu_{0}\right)\right)$ is mapped upon the distance $\boldsymbol{l}$,

$$
0 \leq \boldsymbol{l}=\left(\xi_{2} / 2 \pi\right)-\left(\xi_{1} / 2 \pi\right) \leq 1 .
$$


If $\left(\xi_{2} / 2 \pi\right)-\left(\xi_{1} / 2 \pi\right)=0$, the distance is zero and the two parameter values obviously represent the same tone. If $\left(\xi_{2} / 2 \pi\right)-\left(\xi_{1} / 2 \pi\right)=1$, then the two tones differ by an octave.

The distances obtained in this manner are unhandy small numbers. Thus, by convention, the distance formula is renormalized by the factor 1,200 and is given the name cent (out of 1,200 cent),

$$
\begin{aligned}
\boldsymbol{l}(\text { cent }) & =1,200 \log _{2}\left(\nu_{2} / \nu_{1}\right)=1,200\left(1 / \log _{10} 2\right) \log _{10}\left(\nu_{2} / \nu_{1}\right) \\
& =3,986.31371 \log _{10}\left(\nu_{2} / \nu_{1}\right) .
\end{aligned}
$$

This is the standard formula for the distance (interval) between two musical tones in terms of cent.

Given two musical tones in the form of lattice points,

$$
\left(\nu_{2} / \nu_{0}\right)=(n, m, r) \text { and }\left(\nu_{1} / \nu_{0}\right)=(k, l, u), \quad n, m, r, k, l, u \text { integers, }
$$

the frequency ratio of the two tones is obtained as

$$
\begin{aligned}
\nu_{2} / \nu_{1} & =(2 / 1)^{n}(3 / 2)^{m}(5 / 3)^{r} /(2 / 1)^{k}(3 / 2)^{l}(5 / 3)^{u} \\
& =(n-k, m-l, r-u) .
\end{aligned}
$$

Thus it follows

$$
\begin{aligned}
\log _{2}\left(\nu_{2} / \nu_{1}\right) & =\log _{2}(2 / 1)^{n-k}+\log _{2}(3 / 2)^{m-l}+\log _{2}(5 / 3)^{r-u} \\
& =n-k+(m-l) 1.58496250+(r-u) 2.32192809 .
\end{aligned}
$$

The cent are then given by the formula

$$
\boldsymbol{l}(\text { cent })=1,200(n-k)+701.955500(m-l)+884.35871(r-u) \text {. }
$$

It is thus seen that the cents for the distance between two musical tones are given by the sum of the cents along the (2/1)-based octave, the (3/2)-based octave and the (5/3)-based octave, respectively. The distance (in cent) between two musical tones is thus expressed as a linear equation in terms of three discrete parameters.

\section{Determination of Tones - the Three-Dimensional 116-Tone System}

In this section the Table of Tones, as given in [2], pp. 796-801, is discussed. It will be shown that, by adding a few tones to this list, a structured lattice tone system of 116 tones is obtained which is based upon three intervals (vectors - lattice points) $\lambda, \mu, \rho$ only. 


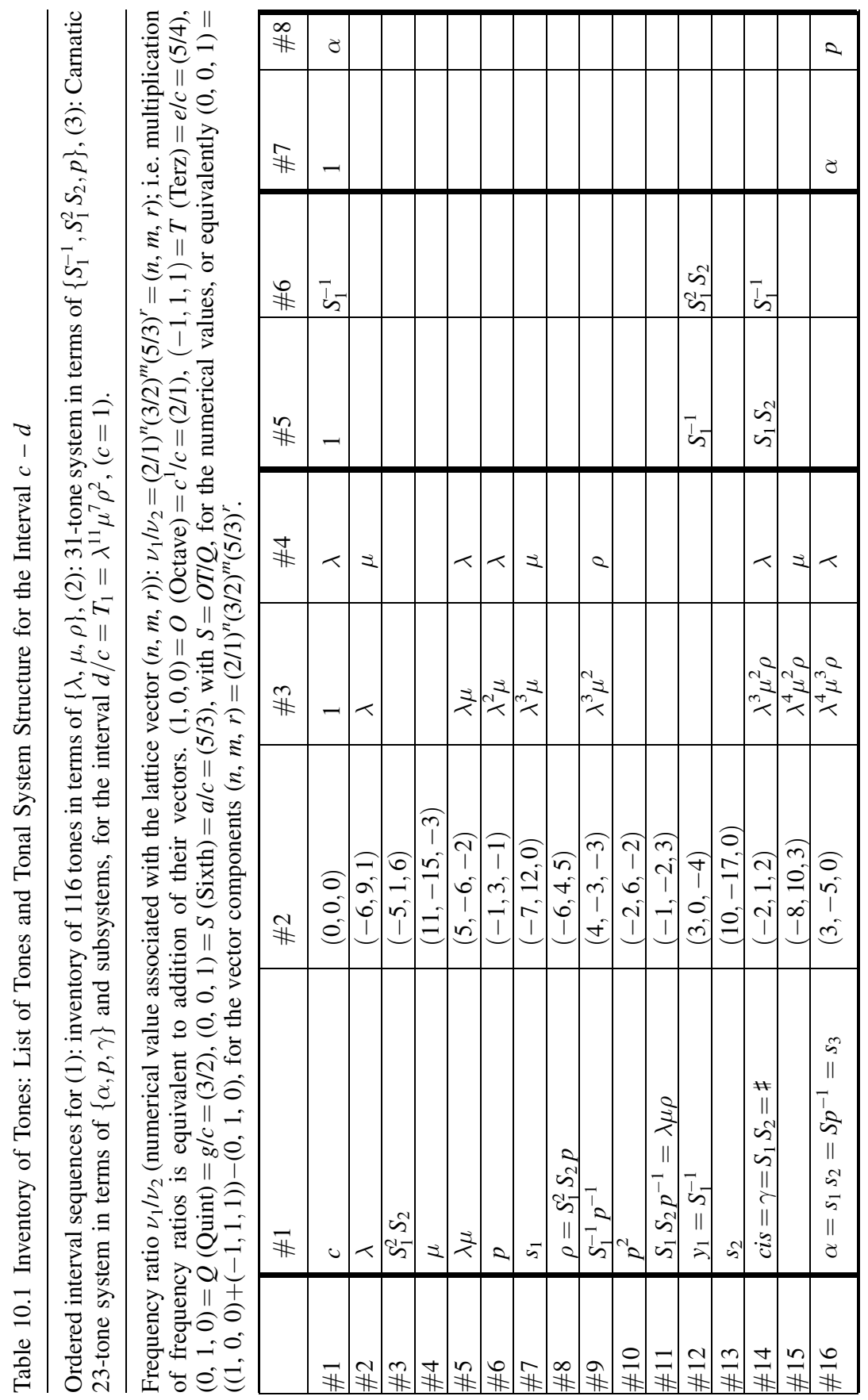




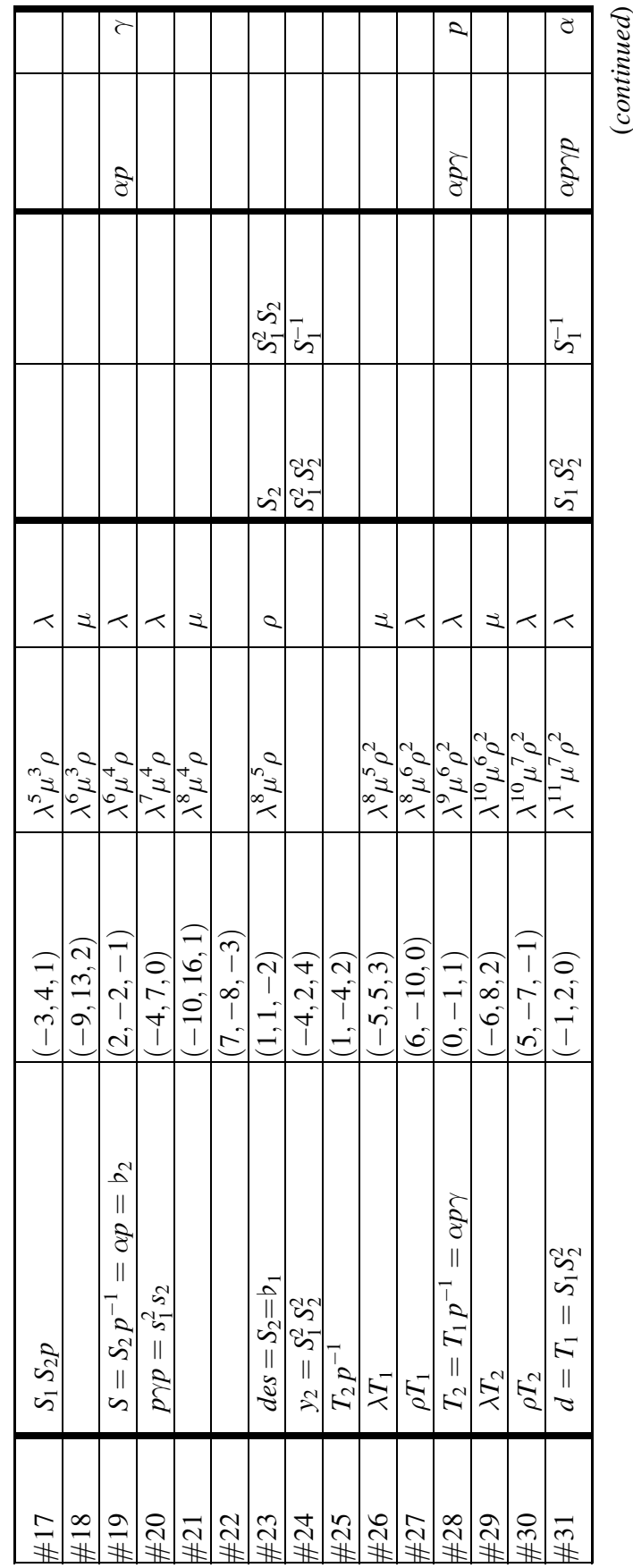




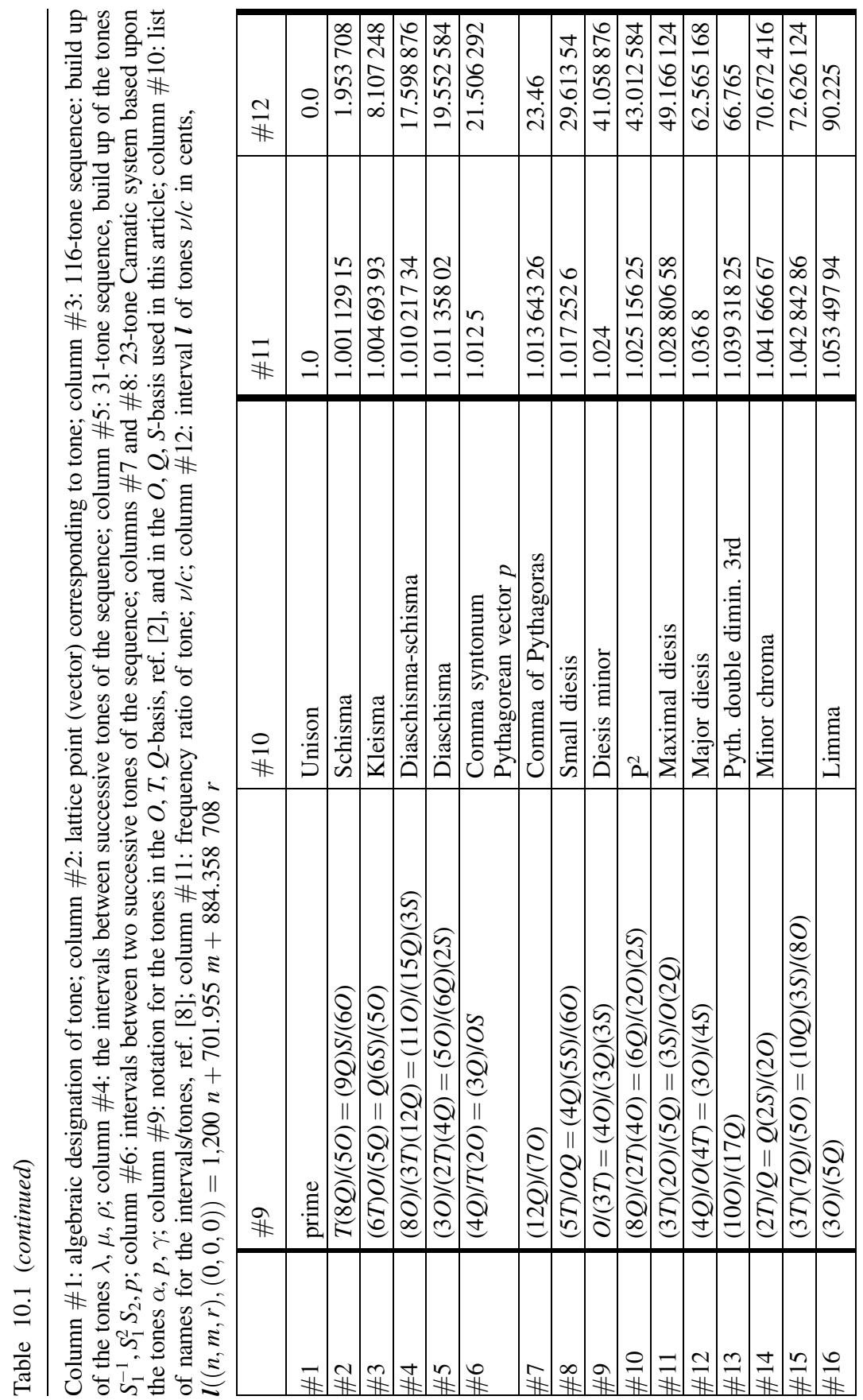




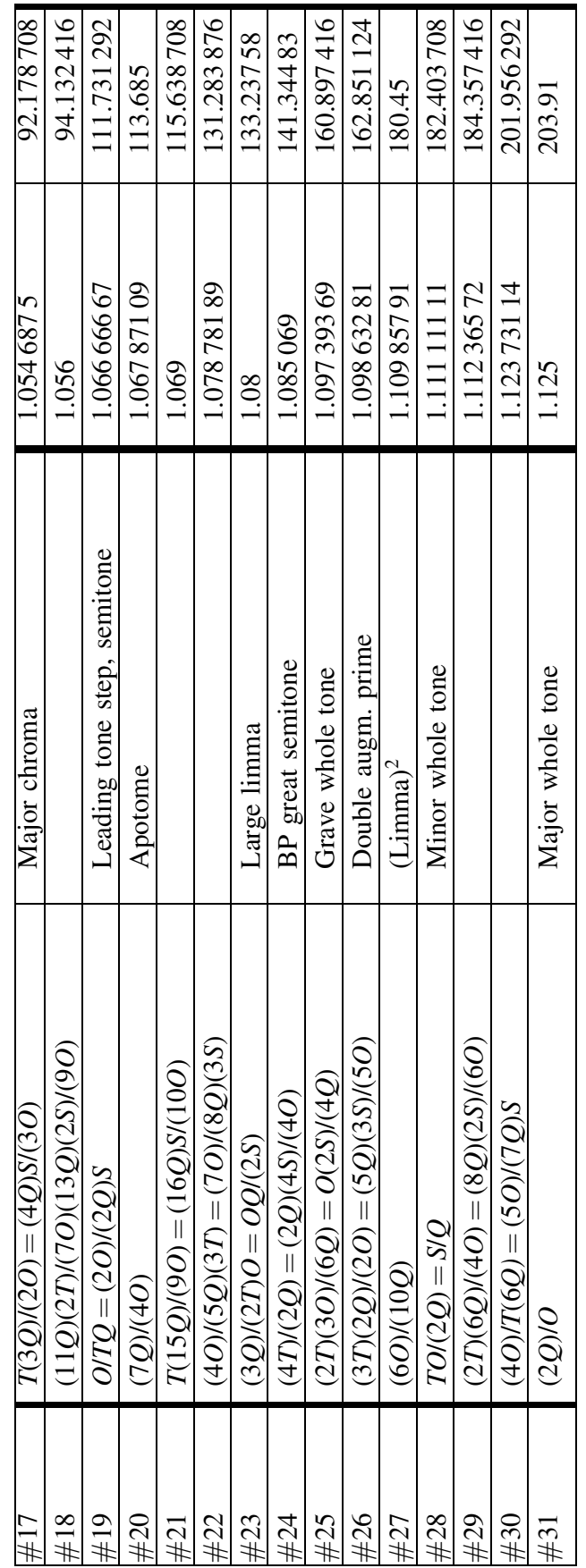


The system of tones, as presented in ref. [2], uses the (musically motivated) basis

$$
\begin{aligned}
& (1,0,0)=O \text { (Octave) }=2 / 1=c^{1} / c, \\
& (-1,1,1)=T \text { (Terz) }=5 / 4=e / c \text {, } \\
& (0,1,0)=Q \text { (Quint) }=3 / 2=g / c,
\end{aligned}
$$

while the (mathematically motivated) basis used in this article is

$$
\begin{aligned}
& (1,0,0)=O \text { (Octave) }=2 / 1=c^{1} / c, \\
& (0,1,0)=Q \text { (Quint) }=3 / 2=g / c, \\
& (0,0,1)=S \text { (Sixth) }=5 / 3=a / c .
\end{aligned}
$$

The two bases are mathematically equivalent,

$$
S=O T / Q \text {. }
$$

Introducing the intervals (tones)

$$
\begin{array}{ll}
\lambda=(-6,9,1) & =T(8 Q) /(5 O), \\
\mu=(11,-15,-3) & =(8 O) /(3 T)(12 Q), \\
\rho=(-6,4,5) & =(5 T) / O Q,
\end{array}
$$

as basis intervals, the closure condition

$$
k_{1} \lambda+k_{2} \mu+k_{3} \rho=(1,0,0)
$$

is satisfied for

$$
k_{1}=63, \quad k_{2}=41, \quad k_{3}=12, \quad N=116 .
$$

Thus a total of 116 tones is obtained.

For purposes of illustration the list of tones for the interval $d / c-c / c$ is given in detail in Table 10.1. For the interval $c-d$ holds $k_{1} \lambda+k_{2} \mu+k_{3} \rho=(-1,2,0), \quad k_{1}=11, \quad k_{2}=7, \quad k_{3}=2, \quad N=20$

and thus the tone interval $c-d$ contains 20 tones. The 30 tones contained in Table 10.1 are then obtained by adding to the list the tones $S_{1}^{2} S_{2}=(-5,1,6), \quad \mu=(11,-15,-3), \quad \rho=(-6,4,5)$, $p^{2}=(-2,6,-2),(-8,15,-1), S_{1}^{-1}=(3,0,-4), s_{2}=(10,-17,0)$, $(-9,13,2),(7,-8,-3), y_{2}=(-4,2,-4)$. Most of these added tones are base intervals for other musical tone systems.

The 20-tone tonal system for the interval $c-d$, that is, for the interval $T_{1}$, is then given, in sequential order, by

$$
\begin{aligned}
T_{1} & =/ \lambda \mu \lambda \lambda \mu \rho \lambda \mu \lambda \lambda \mu \lambda \lambda \mu \rho \mu \lambda \lambda \mu \lambda \lambda / \\
& =S_{3}+S-p .
\end{aligned}
$$


Similarly, for the interval $T_{2}$, the tone interval $d-e$, a 17-tone sequence is obtained as

$$
\begin{aligned}
T_{2} & =/ \lambda \mu \lambda \lambda \mu \rho \lambda \mu \lambda \lambda \mu \lambda \lambda \lambda \mu \rho \mu \lambda / \\
& =S_{3}+S
\end{aligned}
$$

and for the interval $S$, the tone interval $e-f$, an 11-tone sequence is obtained as

$$
S=/ \lambda \mu \lambda \lambda \mu \rho \mu \lambda \lambda \mu \lambda / .
$$

This then yields a 116-tonal system for the octave by means of the (ordered) sequence

$$
/ T_{1} / T_{2} / S / T_{1} / T_{2} / T_{1} / S /
$$

having the octave property

$$
\lambda^{63} \mu^{41} \rho^{12}=2, \quad N=116 .
$$

It needs to be noted that Eqs. (10.8)-(10.10) represent only one particular choice for the sequence of the intervals. This choice for the sequence of intervals has been motivated by the aim to include in a regular manner, as many as possible, of the tones of the List of Tones of [2], and keeping the introduction of new tones to a minimum.

The 116-tone system contains, as sub-systems (Table 10.1),

(a) the tones of the 31-tone system,

(b) the standard chromatic major and minor scales,

(c) the natural diatonic scale,

(d) the tone scale of the ancient Greek Lyre,

(e) the tones of the hypothetical 22/23-tone Carnatic scale,

(f) and the subsystems/scales of all these systems,

(g) the 2-dimensional tonal lattices in the Pythagorean plane by means of projection along the Pythagorean vector into the Pythagorean plane.

Column \#1 of Table 10.1 lists the mathematical-musical designation of the tones. Column \#2 lists the tone by its lattice point coordinates $(n, m, r)$. Column \#3 indicates the cumulative build up of the tones based upon the basis $\lambda, \mu, \rho$. The $\lambda, \mu, \rho$ may be looked upon as frequency ratios or as vectors (for example, the entry $\lambda^{3} \mu^{2}$, can be read as $3 \lambda+2 \mu=3(-6,9,1)+2(11,-15,-3)=(-18,27,3)+$ $(22,-30,-6)=(4,-3,-3))$. Column \#4 shows the interval factor/vector between two neighboring tones. Columns \#5 and \#6 refer to the 31-tone system based upon the base elements $S_{1}^{-1}=(3,0,-4), S_{1}^{2} S_{2}=(-5,1,6)$ and $p=(-1,3,-1)$. Column \#5 
B. J. Gruber
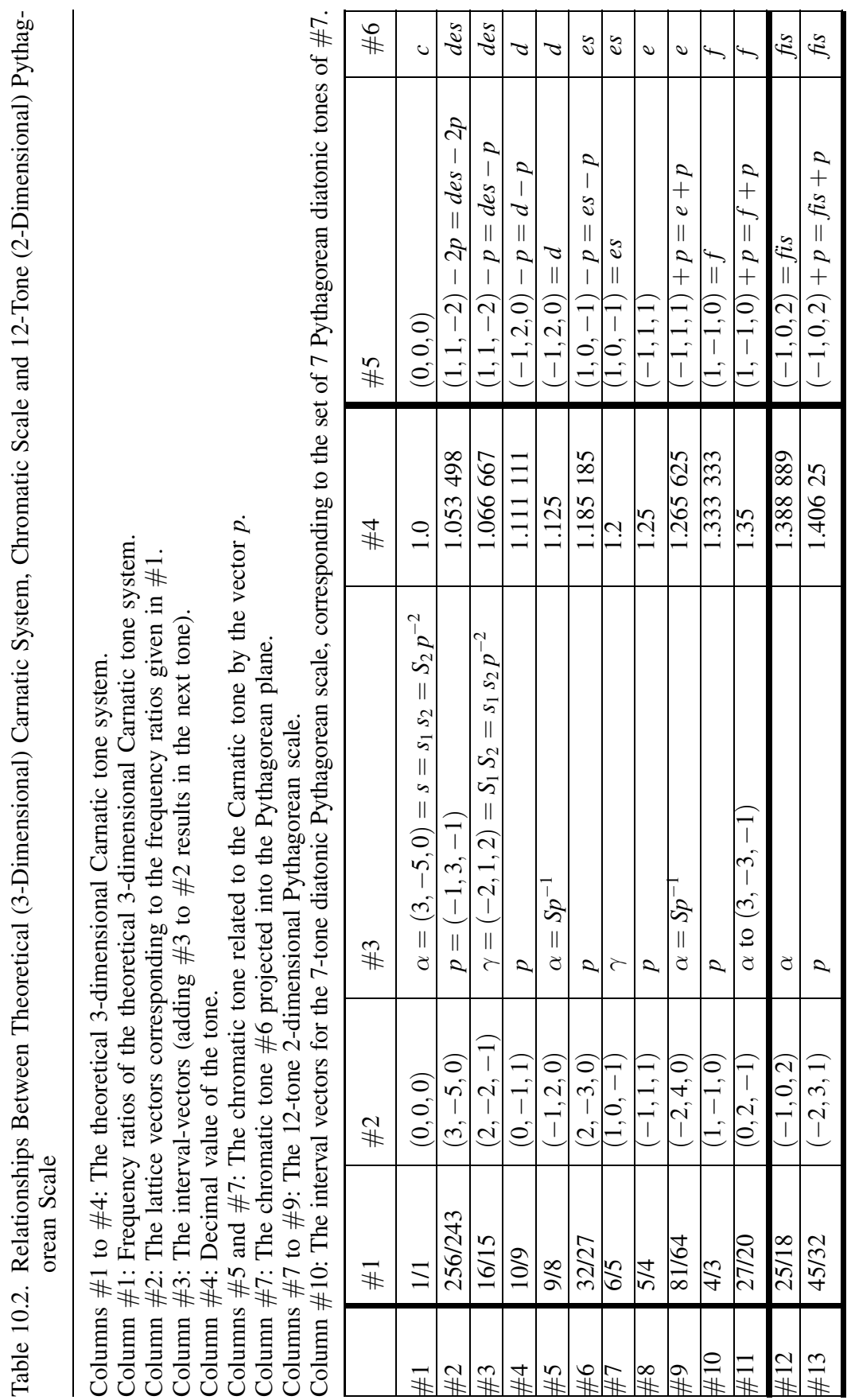


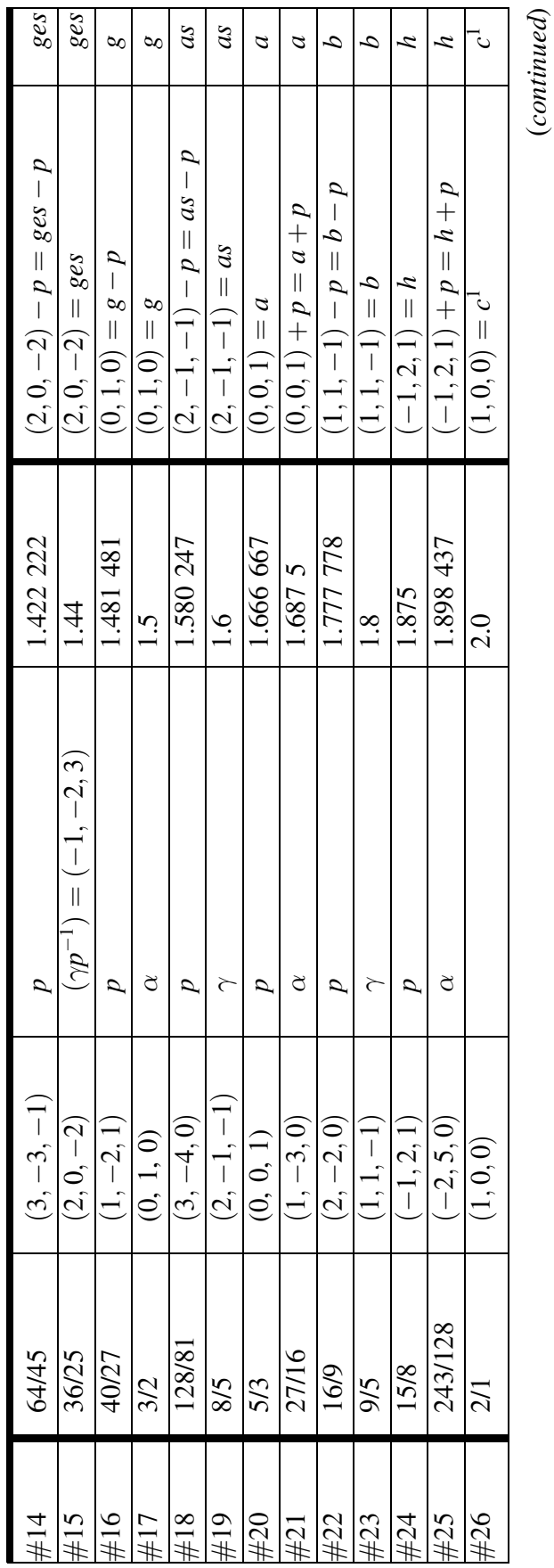




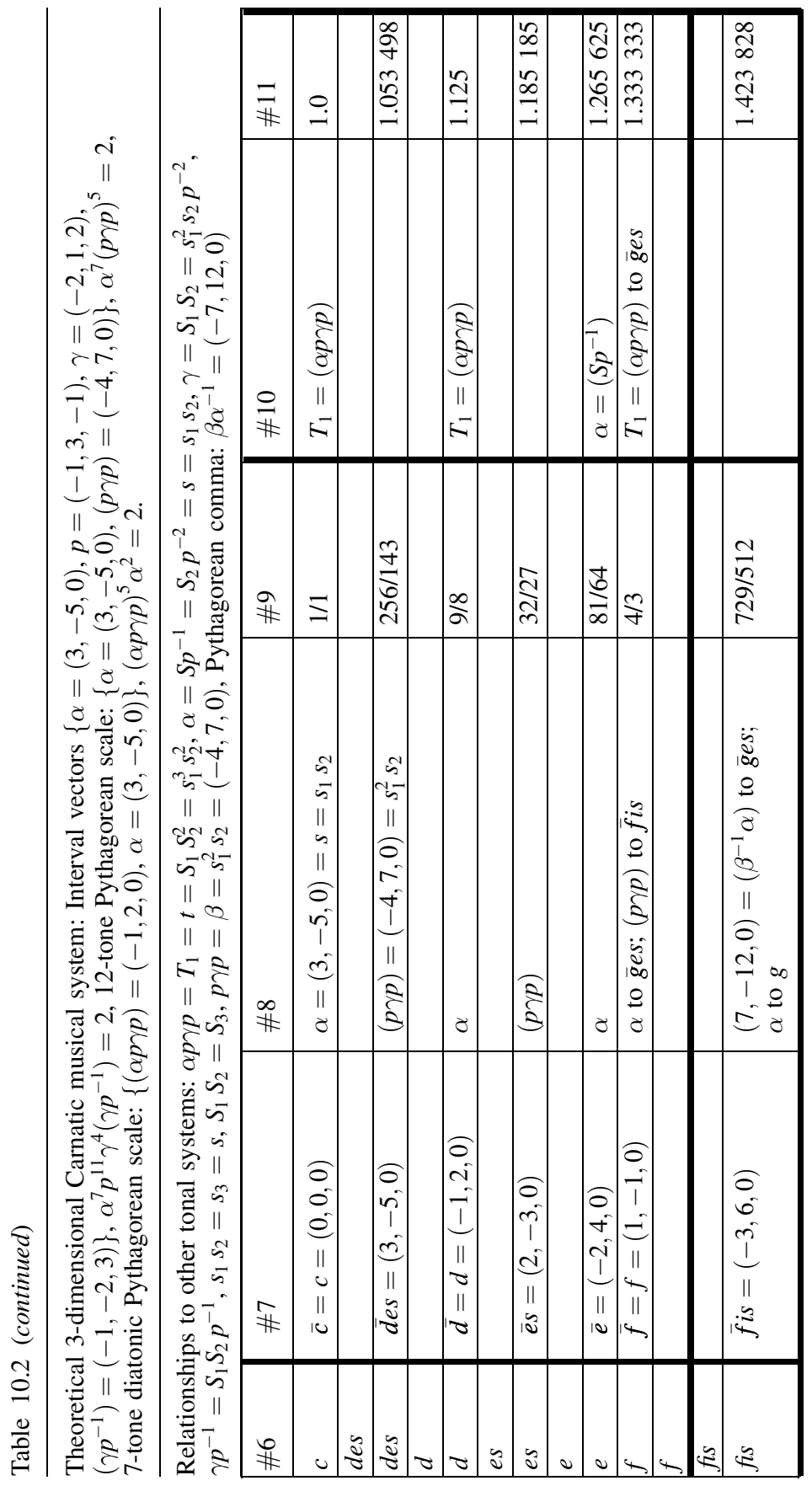




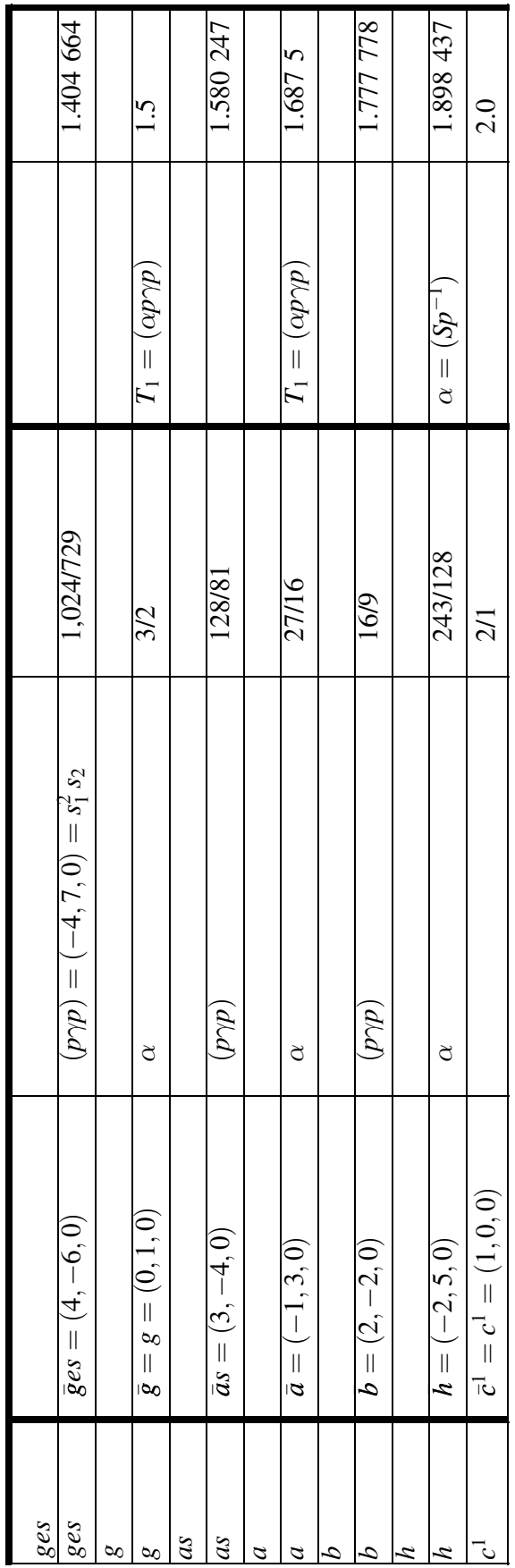


shows the cumulative build up, while column \#6 shows the interval factors/vectors between neighboring lattice tones. Columns \#7 and \#8 describe the hypothetical 23-tone Carnatic system. Column \#7 characterizes the tones, column \#8 lists the interval factors for the basis $\alpha=(3,-5,0), p=(-1,3,-1)$ and $\gamma=(-2,1,2)$. Column \#9 lists the standard musical names for the intervals/tones as given in ref. [12].

The hypothetical 23-tone Carnatic scale consists of the prime $c$ and 11 pairs of tones, with each pair of tones related by the Pythagorean vector $p$. Thus projecting the traditional Carnatic scale along the vector $p$ into the Pythagorean plane 12 tones are obtained. The tone system obtained in this manner corresponds to the 12-tone Pythagorean tone scale, Table 4.1. Table 10.2 contains the following tonal systems:

(a) The traditional 3-dimensional 23-tone Carnatic scale is given by the sequence of intervals

$\left\{\alpha, p, \gamma,\left(\gamma p^{-1}\right)\right\}$,

/ $\alpha p \gamma p / \alpha p \gamma p / \alpha / p \alpha p\left(\gamma p^{-1}\right) p / \alpha p \gamma p / \alpha p \gamma p / \alpha /$, $\alpha^{7} p^{11} \gamma^{4}\left(\gamma p^{-1}\right)=2, \quad N=23$,

$\alpha=(3,-5,0), \quad p=(-1,3,-1), \quad \gamma=(-2,1,2)$,

$(\alpha p \gamma p)=T_{1}=S_{1} S_{2}^{2}=s_{1}^{3} s_{2}^{2}=t, \quad \alpha=S p^{-1}, \quad \alpha^{7} p^{11} \gamma^{4}\left(\gamma p^{-1}\right)=2$.

Note that if in Eq. (10.13) the sequence of intervals $/ p \alpha p\left(\gamma p^{-1}\right) p /$ is taken as $/ p \alpha p \gamma /$ then an $N=22$-tone Carnatic scale/system is obtained. If the order of the sequence in Eq. (10.13) is changed to

$$
\text { / } \alpha p \gamma p / \alpha p \gamma p / \alpha / p\left(\gamma p^{-1}\right) p \alpha p / \alpha p \gamma p / \alpha p \gamma p / \alpha / \text {, }
$$

then the tone $\bar{f} i$ is obtained in place of the tone $\bar{g} e s$. Again, if the sequence $/ p\left(\gamma p^{-1}\right) p \alpha p /$ is replaced by the sequence / $p \gamma \alpha p /$ an $N=22$ Carnatic scale/system is obtained.

(b) The 12-tone Pythagorean scale: The tones obtained via the sequence Eq. (10.14) consist of the prime $c$ and 11 pairs of tones, with each pair related by the Pythagorean vector $p$. Thus, if projected into the Pythagorean plane each pair is mapped upon a single tone. The 12 tones thus obtained correspond to the 12-tone Pythagorean scale given in Table 4.1,

$\{\alpha,(p \gamma p)\}$

$/ \alpha(p \gamma p) / \alpha(p \gamma p) / \alpha / \alpha(p \gamma p) / \alpha(p \gamma p) / \alpha(p \gamma p) / \alpha /$

$\alpha^{7}(p \gamma p)^{5}=2, \quad N=12$, 
$\alpha=(3,-5,0)=S p^{-1}=s_{3}, \quad(p \gamma p)=S_{1} S_{2} p^{2}=(-4,7,0)=\left(s_{1} s_{3}\right)$,

$\gamma p^{-1}=S_{1} S_{2} p^{-1}=(-1,-2,3)$.

See also refs. [10], [11].

(c) The 7-tone natural Pythagorean scale: The Pythagorean 7-tone natural diatonic scale is obtained as

$$
\begin{aligned}
& \left\{T_{1}=t,\left(S p^{-1}\right)=s\right\}, \\
& / T_{1} / T_{1} /\left(S p^{-1}\right) / T_{1} / T_{1} / T_{1} /\left(S p^{-1}\right) /, \\
& T_{1}^{5}\left(S p^{-1}\right)^{2}=2, \quad N=7 .
\end{aligned}
$$

(d) The 3-tone scale of the ancient Greek Lyre:

$$
\left(T_{1} T_{1}\left(S p^{-1}\right)\right) / T_{1} /\left(T_{1} T_{1}\left(S p^{-1}\right)\right), \quad\left(T_{1} T_{1}\left(S p^{-1}\right)\right)^{2} T_{1}=2 .
$$

A summary of the results is given in Table 10.2.

The relationship between the three bases $\{\lambda, \mu, \rho\},\left\{S_{1}^{-1}, S_{1}^{2} S_{2}\right.$, $\left.S_{1}^{-1} p^{-1}\right\}$ and $\{\alpha, p, \gamma\}$ for the 116-tone musical system, the 31-tone musical system and the Carnatic musical system, respectively, is given by

$$
\begin{aligned}
& S_{1}^{-1}=\lambda^{5} \mu^{3}, \quad S_{1}^{-1} p^{-1}=\lambda^{3} \mu^{2}, \quad \lambda=S_{1} p^{3}=s_{1} p^{-1}, \\
& S_{2}=\lambda^{8} \mu^{5} \rho, \quad S_{1}^{2} S_{2}=\lambda^{-2} \mu^{-1} \rho, \quad \mu=S_{1}^{-2} p^{-5}=s_{1}^{-2} p^{3}, \\
& S_{3}=\lambda^{3} \mu^{2} \rho, \quad p=\lambda^{2} \mu, \quad \rho=S_{1}^{2} S_{2} p=s_{1}^{3} s_{2} p^{-5}, \\
& \alpha=S p^{-1}=\lambda^{4} \mu^{3} \rho, \quad T_{1}=(\alpha p \gamma p)=S_{1} S_{2}^{2}=s_{1}^{3} s_{2}^{2}=t, s=s_{3}, \\
& p=S_{2} / S=\lambda^{2} \mu, \quad T_{2}=T_{1} p^{-1}, \\
& \gamma=S_{1} S_{2}=\lambda^{3} \mu^{2} \rho, \quad S=\alpha p .
\end{aligned}
$$

The tones given by Eqs. (10.1) and (10.2) are obtained, in terms of the basic vectors $\lambda, \mu, \rho$, Eq. (10.4), by means of the vector equation

$$
(n, m, r)=k_{1} \lambda+k_{2} \mu+k_{3} \rho, \quad k_{i}, i=1,2,3, \text { integers, }
$$

by substituting for $(n, m, r)$ the desired lattice tone. For the lattice tone $g / c=(0,1,0)$ one obtains the set of three equations

$$
\begin{aligned}
& 0=-6 k_{1}+11 k_{2}-6 k_{3}, \\
& 1=9 k_{1}-15 k_{2}+4 k_{3}, \\
& 0=k_{1}-3 k_{2}+5 k_{3} .
\end{aligned}
$$


These equations yield the values

$$
k_{1}=37, \quad k_{2}=24, \quad k_{3}=7,
$$

and thus

$$
g / c=(0,1,0)=3 / 2=\lambda^{37} \mu^{24} \rho^{7}, \quad N=68 .
$$

Similarly one obtains

$$
\begin{aligned}
a / c & =(0,0,1) & =5 / 3 & =\lambda^{46} \mu^{30} \rho^{9}, & & N=85, \\
e / c & =(-1,1,1) & =5 / 4 & =\lambda^{20} \mu^{13} \rho^{4}, & & N=37, \\
c^{1} / c & =(1,0,0) & =2 / 1 & =\lambda^{63} \mu^{41} \rho^{12}, & N & =116,
\end{aligned}
$$

and

$$
\begin{aligned}
f / c & =\left(c^{1} / c\right)(g / c)^{-1}=\lambda^{63} \mu^{41} \rho^{12}\left(\lambda^{37} \mu^{24} \rho^{7}\right)^{-1}=\lambda^{26} \mu^{17} \rho^{5}, \\
N & =48 .
\end{aligned}
$$

By introducing still finer lattice systems it is possible to approximate arbitrarily close any tonal system (see also ref. [13]), like the 12-tone equal-tempered tonal scale $\left({ }^{12} \sqrt{2}\right)$ or the 25 -tone experimental scale $(25 \sqrt{5})$, developed by STOCKHAUSEN, in terms of "natural parameters (intervals)". While the equal tempered scale is based upon the lattice tone $(1,0,0)=2 / 1$, STOCKHAUSEN's scale is based upon the lattice tone $(1,1,1)=(2 / 1)(3 / 2)(5 / 3)=5$, an octave plus a fifth plus a sixth.

\section{Acknowledgement}

The author wishes to thank Professors Miguel LoRENTE, DiETER FluRY and PETER KRAMER for their interest and their comments.

\section{References}

[1] Gruber, B. J.(2005) Mathematical-Physical Properties of Musical Tone Systems. Sitzungsber. Öst. Akad. Wiss. Wien, math.-nat. Kl., Abt. II 214: 43-79

[2] Riemann, H. (1970) Dictionary of Music, pp. 796-801. Angerer \& Co, London, Da Capo Press, New York (Music Theory Reprint Series)

[3] Mazzola, G. (1990) Geometrie der Töne. Birkhäuser, Boston, Berlin

[4] LORENTE, M. (1964) Fundamentos fisicos de la tonalidad y la consonancia. Rev. Cienc. Aplic. 97: 97-116; LORENTE, M. (1965) Physical basis of tonality and consonance. V. Int. Congress of Acoustics, Liege; FERNANDEZ-HERRERO, O., LORENTE, M. (2006) Comprobación experimental de la teoria de consonancia y disonancia musical. Rev. Acustica 37: 5-10

[5] FluRY, D. (1992) Mathematik als Sprache einer Theorie des Hörbewusstseins. In: Klang und Komponist (BIBA, O., SCHUSTER, W., eds.). Hans Schneider, Tutzing

[6] KishiBE, S. (1984) The Traditional Music of Japan. Ongaku no Tomo-Sha, Tokyo 
[7] Malm, W. P. (1967) Music Cultures of the Pacific, the Near East and Asia (History of Music Series). Prentice Hall, New York

[8] HuYGENS-FOKKER, S.: List of Intervals. http://www.xs4all.nl huygensf/doc/ intervals.html

[9] WADE, B. C. (2004) Music in India - the Classical Traditions. Monahor, New Delhi

[10] Krishnaswamy, A. (2003) On the Twelve Basic Intervals in South Indian Classical Music. Audio Engineering Society Convention Paper \#5903

[11] Sambamurthy, P. (1982) South Indian Music, Vol. 4. The Indian Music Publishing House, Madras

[12] PIERCE, J. R. (1983) The Science of Musical Sound, p. 67 (Scientific American Library). Freeman, New York-San Francisco

[13] See ref. [3], p. 31

Author's address: Prof. Bruno J. Gruber, Visiting Scientist, Institut für Meteorologie und Geophysik, Universität Wien, Hohe Warte 38, 1190 Wien, Austria. Permanent address: Emeritus, College of Science, Southern Illinois University, Mailcode 4403, Carbondale, IL 62901, USA. E-Mail: Gruber@ siu.edu. 